\title{
DETECTION OF INTERMEDIATE VECTOR BOSONS AND HIGH-ENERGY WEAK INTERACTIONS FROM DECAY OF HADRON RESONANCES *
}

\author{
H.E. HABER ${ }^{+}$and G.L. KANE \\ Physics Department, University of Michigan, Ann Arbor, MI 48109, USA
}

Received 1 June 1978

\begin{abstract}
We discuss the detection of parity violating effects in the production of hadron resonances as a way to identify weak interactions at high energies. Numerical results indicate that such effects due to the production of W's and Z's should be observable at Isabelle energies. Observation of such effects may be the best method to establish that high-mass resonances or new behavior of cross sections are not hadronic in origin. By observing hadron resonances with different quantum numbers, it is possible to study the flavor dependence of high-energy weak effects; the energy dependence of the $\varphi / \pi$ ratio may even be a useful signal of $W$ production. We also calculate predictions for polarized beams. The possibility of observing unconventional kinds of weak interactions is briefly considered.
\end{abstract}

\section{Introduction}

In this paper we want to discuss how weak interactions can be studied at high energies. We will concentrate on productions of W's and Z's but also include some discussion of the possibility of seeing unconventional effects. The usual methods [1-3] involve the production of W's and Z's and their decays into leptons or hadron jets. In particular, the charge asymmetry in $\mathrm{pp} \rightarrow \mu^{+} \mu^{-}+\mathrm{X}$, or the frontback asymmetry in $\mathrm{p} \overline{\mathrm{p}} \rightarrow \mu^{ \pm}+\mathrm{X}$ would signal a weak-interaction effect [3]. These may well be the best methods, particularly to detect a $Z^{0}$ by its $\mu^{+} \mu^{-}$decay, and they have been studied in some detail. The method we want to emphasize here is the detection of weak effects via the parity-violating production of hadron resonances.

One can see very simply that such effects may occur. For example, a $W$ decay into $q \bar{q}$ will give a left-handed $q$ in a $V-A$ theory. When making a meson resonance by picking up a $\bar{q}$ from the vacuum it will form (say) $\rho$ 's only of helicity 0 and -1 , while the strong interactions give equal amounts of helicity 1 and -1 . Thus, appropriate comparison of the amounts of helicity 1 and $-1 \rho$ 's can signal a

\footnotetext{
* Research supported by the Department of Energy.

+ Present address: Theoretical Physics Group, Lawrence Berkeley Laboratory, Berkeley, CA. 94720, USA.
} 
weak effect. Of course, confinement mechanisms could reduce or eliminate such effects; we assume that quark fragments with spin will remember the quark polarization.

While the possibility of detecting weak effects by such a method has been mentioned [4,5], it appears not to have been studied in any detail, so we have performed fairly extensive calculations. We do not mean to argue that this method is better than or even as good as the usual ones. However, the numerical results are encouraging and one can find situations where background effects and experimental considerations appear to favor hadron resonance detection over other methods. Further, the method does seem to have two unique and potentially powerful advantages:

(i) by detecting parity violation one can determine experimentally that a detected resonance or large effect is due to weak interactions;

(ii) by detecting resonances with different charge, strangeness, etc., one can study the flavor dependence of the $\mathrm{W}$ and $\mathrm{Z}$ couplings and of high-energy weak effects in general.

For completeness we also calculate effects expected with polarized beams for total and inclusive cross sections. Apparently such beams may be possible [6] at the next generation of colliding beam machines.

We calculate in some detail the parity-violating effects expected from different $\mathrm{W}, \mathrm{Z}$ spectra. For example, the effects are significantly different in an $\mathrm{SU}(2) \otimes \mathrm{U}(1)$ theory with a single W and in an $\mathrm{SU}(2)_{\mathrm{L}} \otimes \mathrm{SU}(2)_{\mathrm{R}} \otimes \mathrm{U}(1)$ theory with two W's separated somewhat in mass. We also discuss the possibility of detecting unconventional and unexpected weak-interaction effects and distinguishing these from expected ones, because some kinds of behavior would not arise from any simple set of intermediate vector bosons.

In sect. 2, we describe the calculations used to estimate the size of the effect expected in different situations, and give several results relevant to colliding beam $\overline{\mathrm{p}} \mathrm{p}$ and $\mathrm{pp}$ machines. Sect. 3 gives some results for collisions with polarized beams. In sect. 4 , we briefly discuss the detection of unconventional weak-interaction effects. Details of the calculations are gathered into an appendix so that interested readers can reproduce our results.

\section{Detection of weak effects via parity-violating decays of hadron resonances}

\subsection{General remarks}

As we remarked in sect. 1, it appears that a potentially useful method of detecting weak-interaction effects at high energies (including W's and Z's) is to measure parity-violating effects in the decay of hadron resonances such as $\mathrm{K}^{*}, \varphi, \rho, \Delta, \Lambda$, etc. This method does not seem to have been studied in detail, so we have carried out calculations to study how useful it might be. 
It turns out that the method may be of significant value for three reasons. First, we find that except at very large $p_{\mathrm{T}}$, the expected rate for producing a hadron resonance dominates the rate of single-lepton production. This occurs because, whereas the quark (or lepton) which emerges is very energetic, the hadron originating from the fragmenting quark tends to come out at lower $p_{\mathbf{T}}$. Hence, the emerging hadron contains information about the polarization of a quark of momentum larger than its own. Second, if the method works, it can be used to study the quantum numbers of weak effects by comparing the results for different resonances. Third, it directly signals a weak interaction.

One caveat is of course needed. No one knows how quarks materialize into hadron resonances, or how much of the quark polarization will be remembered by a resonance. We are presuming that a substantial fraction of the quark polarization is retained by the hadron. One can think in two stages; first comes finding the effect at all, which could be enough to detect W's and verify that they are objects with weak interactions. Then comes interpreting the specific effect to deduce the actual properties of the weak interaction. It is reasonable to be optimistic that enough will be learned about the parton model in the next few years to expect to be able to interpret an effect if one is found. It should be noted that the one available indication, the asymmetry in polarized electroproduction [7], is consistent with the assumption that quark polarizations are remembered by hadrons.

A useful check would be to find hadron resonances in $\nu$ reactions where it is known that there is a polarized quark and determine if such resonances show effects of their weak production. Unfortunately, as we will see below, detection of parity violation requires both that helicities be populated asymmetrically and that the appropriate amplitudes be out of phase. In hadron reactions (such as high-energy colliding beams), in general, the weak and strong production will be out of phase and will produce an observable effect, while in $\nu$ reactions different helicities will be present but the purely weak production may not give large enough phase differences to see an effect. It is still worthwhile to look for a positive effect in $\nu$ reactions; for example, because of their parity-violating decays, $\Lambda$ 's do not require the phase difference (a non-zero $p_{z}$ associated with $a \cos \theta$ in the decay angular distribution should be present). Also, interference of resonance production and background in the hadron system can give the needed phase difference; weak production of $\Delta$ 's in $\nu \mathrm{p} \rightarrow \mu^{-} \Delta^{++}$has recently been observed [8] this way.

\subsection{Description of calculations}

We want to estimate in a model calculation the weak-interaction contribution to inclusive production of hadron resonances. We examine the energy regime above the W-boson threshold. This region is not accessible to present day accelerators, but will be attainable at proposed $\bar{p} p$ colliding machines at CERN [9] and Fermilab [10], and at Isabelle.

If only conventional weak effects were to occur at such energies, then the weak- 


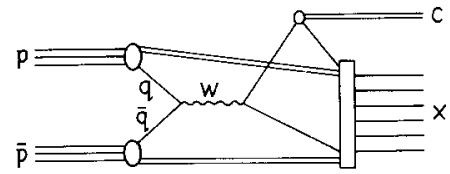

Fig. 1. Weak interaction contribution to $\mathrm{p} \overline{\mathrm{p}} \rightarrow \mathrm{C}+\mathrm{X}$. A quark and anti-quark annihilate into a real $\mathrm{W}$-boson. The $\mathrm{W}$-boson then decays into the observed hadron $\mathrm{C}$ plus other unobserved particles.

interaction contribution which could be detected would involve the production of weak vector bosons (and subsequent decay into hadron resonances). We will focus our attention on this contribution to inclusive hadron scattering. Our aim is to demonstrate that parity violation associated with W's and Z's is observable. Once the properties of the weak vector bosons are well-understood, one will be able to regard the W's and Z's as experimental background to other weak-interaction phenomena. The hope is that if unconventional weak effects existed, they would be identified by observable parity violation not attributable to $\mathrm{W}$ and $\mathrm{Z}$ production.

We wish to calculate the cross section for the processes:

$$
\begin{aligned}
& \mathrm{pp} \rightarrow \mathrm{C}+\mathrm{X}, \\
& \overline{\mathrm{p}} \mathrm{p} \rightarrow \mathrm{C}+\mathrm{X},
\end{aligned}
$$

where $\mathrm{C}$ results from the decay of a weak vector boson ${ }^{\star}$. We use the quark-quark scattering model of Berman, Bjorken, and Kogut (BBK) [11]. This is pictorially described in fig. 1. A quark and antiquark from the beam annihilate into a weak vector boson which subsequently decays into quarks. Finally, one of the quarks fragments into the observed hadron $\mathrm{C}$.

In recent years, many authors [12-14] have used the BBK [11] framework to perform calculations of both strong- and weak-interaction phenomena. Several of these calculations have become quite sophisticated. However, there are a number of reasons why we feel that a crude calculation is appropriate here at the present time. First, we are mostly after qualitative results, e.g., whether inclusive resonance production can be useful in studying weak-interaction effects at very large energies. Second, a lot of the fine details that some authors have used are not settled, and ideas are likely to change (or be discovered) during the next few years. And third, certain quantities (such as polarized quark functions) that are not well-known presently will be studied carefully in experiments now in progress. Given these reasons, we feel justified in making many simplifying approximations in the course of our calculations.

* Of course, we are equally interested in $C$ produced by any other weak process. However, as discussed below, weak non-resonant $\mathrm{q} \overline{\mathrm{q}}$ or qq scattering gives effects small compared to the ones we calculate. If effects are observed, their dependence on kinematic variables can be used to check any given interpretation. 
In the appendix, we have provided a detailed account of the BBK formalism as applied to the weak-interaction contribution and the approximations that we have made. Here we will briefly display the basic formulas used in obtaining the results to be given in subsect. 2.3.

Consider the reaction $\mathrm{A}+\mathrm{B} \rightarrow \mathrm{C}+\mathrm{X}$ which proceeds via the production of a positively charged weak vector boson (with mass $m_{\mathrm{V}}$ and width $\Gamma$ ). A and B are assumed to be non-strange hadrons. The required changes for other charge states will be obvious. Suppose that this vector boson couples to quarks by $g \gamma_{\mu}\left(g_{\mathrm{V}}+g_{\mathrm{A}} \gamma_{5}\right)$, where $g_{\mathrm{V}}=g_{\mathrm{A}}=1$ corresponds to pure $\mathrm{V}-\mathrm{A}$ and $g^{2} / m_{\mathrm{W}}^{2} \equiv G_{\mathrm{F}} / \sqrt{ } 2$. Then we find:

$$
\begin{aligned}
E \frac{\mathrm{d}^{3} \sigma}{\mathrm{d} p^{3}} & =\frac{1}{\pi} \frac{\mathrm{d}^{2} \sigma}{\mathrm{d} y \mathrm{~d} p_{\mathrm{T}}^{2}} \\
= & \frac{G_{\mathrm{F}}^{2} m_{\mathrm{W}}^{4}}{2 \pi \Gamma m_{\mathrm{V}} s} \sum_{\mathrm{q}_{2}, \overline{\mathrm{q}}_{1}}\left\{\frac{1}{x_{1}^{2}} \int_{x_{\min }}^{x_{\max }} \frac{x\left[C_{1}+C_{2} \xi^{2}\right]}{(1+\xi)^{4}}\right. \\
& \quad \times\left[u^{\mathrm{A}}(x) \bar{d}^{\mathrm{B}}\left(m_{\mathrm{V}}^{2} / x s\right) G_{\mathrm{q}_{2} \mathrm{C}}(z)+\bar{d}^{\mathrm{A}}(x) u^{\mathrm{B}}\left(m_{\mathrm{V}}^{2} / x s\right) G_{\overline{\mathrm{q}}_{1} \mathrm{C}}(z)\right] \mathrm{d} x \\
& \left.+\left(x_{1} \leftrightarrow x_{2}, \mathrm{~A} \leftrightarrow \mathrm{B}\right)\right\},
\end{aligned}
$$

where the sum is over all charge $\frac{2}{3}$ quarks $q_{2}$ and charge $\frac{1}{3}$ antiquarks $\bar{q}_{1}$ which will fragment into hadron $C$. The variables above are defined as follows: $x_{1}=p_{\mathrm{T}} \mathrm{e}^{y} / \sqrt{ } s$, $x_{2}=p_{\mathrm{T}} \mathrm{e}^{-y} / \sqrt{ } s, \xi \equiv x^{2} x_{2} s / x_{1} m_{\mathrm{V}}^{2}$, and $z=x_{1}(1+\xi) / x . C_{1}$ and $C_{2}$ depend on $g_{\mathrm{V}}$ and $g_{\mathrm{A}}$; they are defined by eq. (A.13). The limits of integration $x_{\min }$ and $x_{\max }$ depend on the values of $x_{1}$ and $x_{2}$ as discussed in the appendix (cf. eq. (A.22)). The quark function $u^{\mathrm{A}}(x)$ is the probability of finding a u-quark in A, etc. In this paper, we approximate the Cabibbo angle to be zero, and we will neglect the strange quarks (as well as heavier quarks) in $\mathbf{A}$ and $\mathbf{B}$; in our kinematic regions these are good approximations.

Eq. 2 (for a given $\bar{q}_{1}, q_{2}$ ) is a sum of four terms corresponding to the four diagrams of fig. 2. The first two terms correspond to diagrams (a) and (b); the last two terms (obtained from the first two by interchanging $x_{1} \leftrightarrow x_{2}$ and $\mathrm{A} \leftrightarrow \mathrm{B}$ ) correspond to diagrams (c) and (d). For later convenience, we will write

$$
\frac{1}{\pi} \frac{\mathrm{d}^{2} \sigma}{\mathrm{d} y \mathrm{~d} p_{\mathrm{T}}^{2}}=I_{\mathrm{a}}+I_{\mathrm{b}}+I_{\mathrm{c}}+I_{\mathrm{d}},
$$

where $I_{\mathrm{x}}$ corresponds to the contribution of diagram $\mathrm{x}$. A similar result holds for the case of $\mathrm{W}^{-}$exchange if we interchange the role of the charge $\frac{2}{3}$ and charge $-\frac{1}{3}$ quarks. We will denote the four terms thus obtained by $I_{\mathrm{e}}, I_{\mathrm{f}}, I_{\mathrm{g}}$ and $I_{\mathrm{h}}$. Note that if $I_{\mathrm{a}}$ appears in a cross section formula (similar to eq. (3)) for a particular process, then so does $I_{c}$. This is true because both diagrams (a) and (c) of fig. 2 represent a hadron $\mathrm{C}$ which results from the decay of the same quark. Similar conclusions can 
(a)

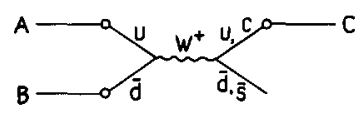

(b)

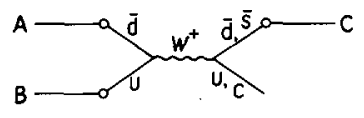

(c)

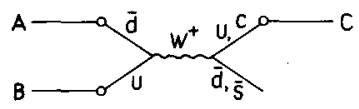

(d)

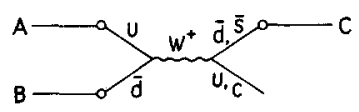

Fig. 2. Inclusive production of $\mathrm{C}$ via s-channel $\mathrm{W}^{+}$exchange. Assume that $\mathrm{A}$ and $\mathrm{B}$ are nonstrange hadrons. We ignore heavy quarks in the sea and assume zero Cabibbo angle. $\mathrm{A} \mathrm{W}^{+}$boson must then originate from $u \bar{d}$ annihilation. The u-quark can either come from A or B; hadron C can emerge either from a charge $\frac{2}{3}$ quark or a charge $\frac{1}{3}$ antiquark. Hence, there are four possible diagrams.

be made of the other three pairs of $I_{\mathbf{x}}$; hence we define:

$$
\begin{array}{ll}
I_{1}=I_{\mathrm{a}}+I_{\mathrm{c}}, & I_{2}=I_{\mathrm{b}}+I_{\mathrm{d}}, \\
I_{3}=I_{\mathrm{e}}+I_{\mathrm{g}}, & I_{4}=I_{\mathrm{f}}+I_{\mathrm{h}} .
\end{array}
$$

Notice that we have omitted contributions from $t$ - and $u$-channel $\mathrm{W}^{ \pm}$exchanges in this discussion. We can do this because we are interested in the kinematic region above $\mathrm{W}$ threshold such that the W's in fig. 2 are produced on-mass-shell. The BreitWigner resonance denominator leads to an enhancement factor which explains why the s-channel $W^{ \pm}$exchange dominates all other weak effects in both $\mathrm{pp}$ and $\mathrm{p} \overline{\mathrm{p}}$ scattering.

We are also interested in the cross section for the production of resonances in a definite helicity state. The required modification of eq. (2) is discussed in subsect. A.5 of the appendix. For the special case of W exchange $\left(g_{\mathrm{V}}=g_{\mathrm{A}}=1\right)$, the cross section formulas become fairly simple. This happens because of the simple model we have used for $G_{\mathrm{q}_{\lambda} \mathrm{C}_{\lambda^{\prime}}}(z)$ (where q is a quark of helicity $\lambda$ which fragments into hadron $C$ of helicity $\left.\lambda^{\prime}\right)$. As discussed in subsect. A.4 of the appendix, we have written

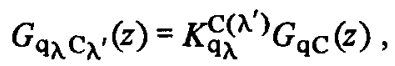

where $K$ is some constant determined from the $\mathrm{SU}(6)$ wave function for $\mathrm{C}\left(\lambda^{\prime}\right)$ and 
Table 1

Cross section for the inclusive production of polarized hadrons from $\mathrm{W}$ decay

\begin{tabular}{lcl}
\hline Hadron & Helicity & Cross section \\
\hline \multicolumn{2}{l}{$(1)$ Definite } & helicity states \\
$\rho^{+}$ & +1 & $0.2 I_{2}$ \\
& -1 & $0.2 I_{1}$ \\
$\omega^{0}$ & +1 & $0.1\left(I_{2}+I_{4}\right)$ \\
& -1 & $0.1\left(I_{1}+I_{3}\right)$ \\
$\mathrm{K}^{*+}$ & +1 & $0.2 I_{2}$ \\
& -1 & $0.1 I_{1}$ \\
$\varphi^{0}$ & +1 & $0.1 I_{2}$ \\
& -1 & $0.1 I_{3}$ \\
$\Lambda^{0}$ & $+\frac{1}{2}$ & $0.005\left(I_{1}+I_{3}\right)$ \\
& $-\frac{1}{2}$ & $0.005\left(I_{1}+5 I_{3}\right)$
\end{tabular}

(2) Average over helicities

$\begin{array}{ll}\rho^{+} & 0.3\left(I_{1}+I_{2}\right) \\ \omega^{0} & 0.15\left(I_{1}+I_{2}+I_{3}+I_{4}\right) \\ \mathrm{K}^{*+} & 0.15\left(I_{1}+2 I_{2}\right) \\ \varphi^{0} & 0.15\left(I_{2}+I_{3}\right) \\ \Lambda^{0} & 0.01\left(I_{1}+3 I_{3}\right)\end{array}$

The $I_{i}$ are defined by eq. (4) in the text. We have assumed that $G_{\mathrm{q} \lambda} \mathrm{C}_{\lambda^{\prime}}(z)=K_{\mathrm{q} \lambda}^{\mathrm{C}\left(\lambda^{\prime}\right)} G_{\mathrm{qC}}(z)$; see subsect. A.4 of the appendix.

$G_{\mathrm{qC}}$ is the unpolarized quark dissociation function. Since the W decays into lefthanded quarks and right-handed antiquarks, it follows that the cross section for $\mathrm{A}+\mathrm{B} \rightarrow \mathrm{C}\left(\lambda^{\prime}\right)+\mathrm{X}$ via $\mathrm{W}^{+}$exchange can be written as a linear combination of the $I_{i}$ (defined by eq. (4)). Therefore, if we assume only W exchange, then the cross sections for many reactions can be written as linear combinations of the $I_{i}$ 's. We list some examples in table 1.

\subsection{Results}

We have calculated the weak-interaction contribution to inclusive resonance production at very high energy. The energy range we have in mind is $100 \leqslant E_{\mathrm{b}} \leqslant$ $1000 \mathrm{GeV}$ where $E_{\mathrm{b}}$ is the energy of each of the colliding beams (i.e., $E_{\mathrm{b}}=\frac{1}{2} \sqrt{s}$ ). In order to determine experimentally that a weak-interaction effect is being seen, it is sufficient to identify some parity violation.

Let us discuss the anticipated strong interaction "background". In fig. 3, we present a (rough) extrapolation of the single $\pi$ inclusive cross section at $E_{\mathrm{b}}=100$ and $400 \mathrm{GeV}$. This extrapolation has been obtained using the results of Field [15]. 


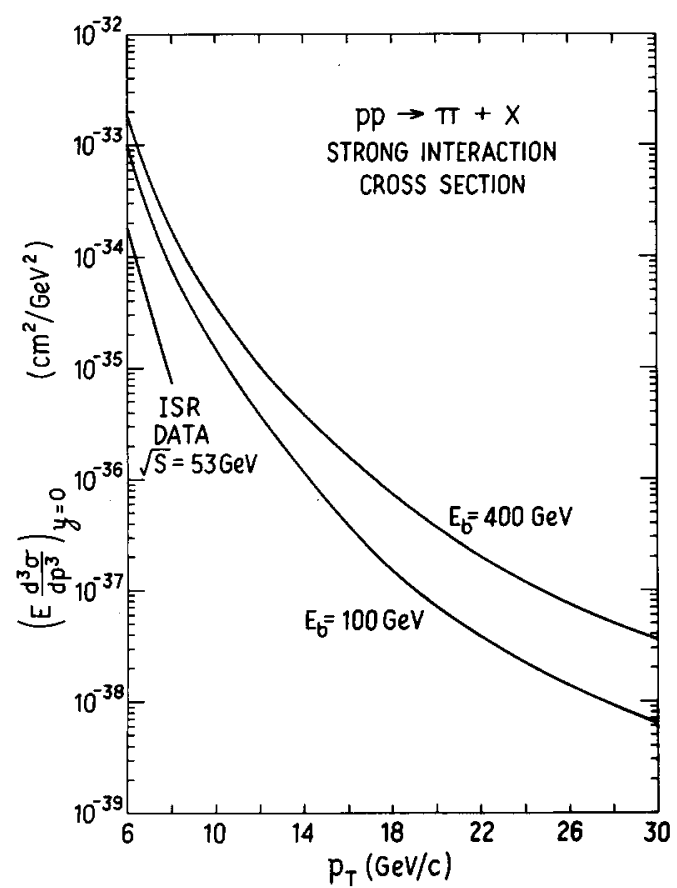

Fig. 3. Extrapolated strong interaction cross section for $p p \rightarrow \pi+X$. The ISR data at $\sqrt{ } s=53$ $\mathrm{GeV}$ comes from ref. [44]. The two extrapolated curves are based on estimates by Field [15]. Note that $E_{\mathrm{b}}=\frac{1}{2} \sqrt{ } s$.

At fixed $x_{\perp}=2 p_{\mathrm{T}} / \sqrt{ } s$, Field expects

$$
\left.E \frac{\mathrm{d}^{3} \sigma}{\mathrm{d} p^{3}}\right|_{\substack{90^{\circ} \\ \text { fixed } x_{\perp}}} \sim \frac{1}{p_{\mathrm{T}}^{6}},
$$

for $p_{\mathbf{T}}>10 \mathrm{GeV} / c$. We have also used [16],

$$
\left.E \frac{\mathrm{d}^{3} \sigma}{\mathrm{d} p^{3}}\right|_{\substack{90^{\circ} \\ \text { fixed } p_{\mathrm{T}}}} \sim\left(1-x_{1}\right)^{9}
$$

to extrapolate (at fixed $p_{\mathrm{T}}$ ) in energy. The $p_{\mathrm{T}}^{-6}$ behavior is obtained in a hardscattering model based on quantum chromodynamics with scale-breaking quark functions and a fairly large transverse-momentum spread for the parton wave functions. If we assume the traditional $p_{\mathrm{T}}^{-4}$ dependence [11], then we find that the hadronic background would swamp any weak-interaction signal. On the other hand, $p_{\mathrm{T}}^{-8}$ is likely to be an overly optimistic guess. In any case, we feel that the use of 


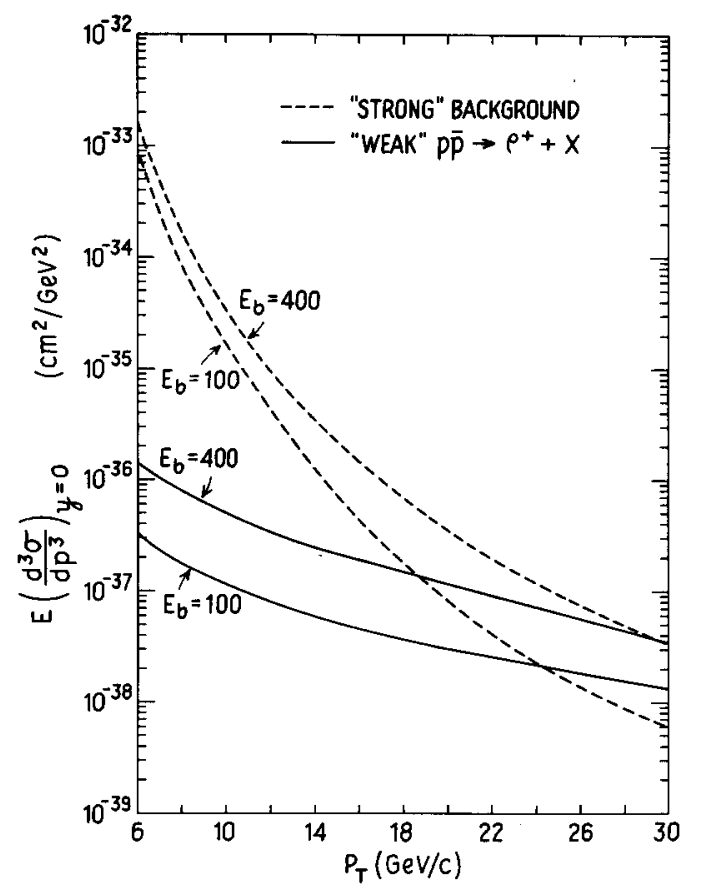

Fig. 4. "Weak" inclusive $\rho^{+}$production and the "strong" background. The "strong" background is just the extrapolated strong interaction cross sections of fig. 3. Current data [17] suggests that the strong interaction inclusive $\pi$ and $\rho$ cross sections at large $p_{\mathrm{T}}$ are about equal.

Field's results is sufficiently realistic for our purposes.

Current data [17] suggest that the inclusive production of $\pi$ and $\rho$ are about equal. (An SU(6) argument would suggest the ratio of $\pi / \rho$ should be $\frac{1}{3}$. However, the inclusive $\pi$ cross section quoted refers not only to prompt pions but all pions produced at a given angle and $p_{\mathrm{T}}$.) If we regard fig. 3 as an approximation to the $\rho$ inclusive cross section, we can compare this with our calculation of $\rho$ production via weak interactions. We wish to determine whether this weak interaction signal could be detected and separated from the large hadronic background. There are two parts to this question. First, we must look for ways to enhance the weak signal relative to background. Second, given realistic luminosities for the next generation of colliding beam machines, we must examine whether the event rate will be sufficient to see any effect at all.

Consider $\overline{\mathrm{p}} \mathrm{p} \rightarrow \rho^{+}+\mathrm{X}$, where the $\rho^{+}$results from the production of a $\mathrm{W}^{+}$. In fig. 4 we compare this with the strong-interaction background at $E_{\mathrm{b}}=100$ and 400 $\mathrm{GeV}$. We note that the weak-interaction signal is swamped by the background for $p_{\mathrm{T}} \leqslant 10 \mathrm{GeV} / c$. It is clear that in order to have a chance to see the weak signal, one must integrate the cross section over the large- $p_{\mathrm{T}}$ region and cut out the small- $p_{\mathrm{T}}$ 
region. This means of course that the event rate will be very small; high luminosity machines will be required.

To get a feeling for the numbers involved, let us define:

$$
\left.\frac{\mathrm{d} \widetilde{\sigma}}{\mathrm{d} y}\right|_{y=0}=\left.\int_{p_{\mathrm{T}}^{\min }}^{\mathrm{m}_{\mathrm{W}} / 2} E \frac{\mathrm{d}^{3} \sigma}{\mathrm{d} p^{3}}\right|_{90^{\circ}} 2 \pi p_{\mathrm{T}} \mathrm{d} p_{\mathrm{T}} .
$$

First, let us choose $p_{\mathrm{T}}^{\min }=10 \mathrm{GeV} / c$. From fig. 4 , we can integrate to obtain:

$$
\left.\frac{\mathrm{d} \widetilde{\sigma}_{\rho}^{\text {background }}}{\mathrm{d} y}\right|_{y=0}=\left\{\begin{array}{lll}
2 \times 10^{-33} \mathrm{~cm}^{2}, & E_{\mathrm{b}}=100 \mathrm{GeV}, & p_{\mathrm{T}}>10 \mathrm{GeV} / c, \\
4.5 \times 10^{-33} \mathrm{~cm}^{2}, & E_{\mathrm{b}}=400 \mathrm{GeV}, & p_{\mathrm{T}}>10 \mathrm{GeV} / c,
\end{array}\right.
$$

which should be compared with the signal we are attempting to see:

$$
\left.\frac{\mathrm{d} \widetilde{\sigma}_{\rho}^{\text {weak }}}{\mathrm{d} y}\right|_{y=0}=\left\{\begin{array}{lll}
10^{-34} \mathrm{~cm}^{2}, & E_{\mathrm{b}}=100 \mathrm{GeV}, & p_{\mathrm{T}}>10 \mathrm{GeV} / c, \\
3.5 \times 10^{-34} \mathrm{~cm}^{2}, & E_{\mathrm{b}}=400 \mathrm{GeV}, & p_{\mathrm{T}}>10 \mathrm{GeV} / c .
\end{array}\right.
$$

Next consider the $y$ dependence in the vicinity of $y=0$ (say for $-2 \leqslant y \leqslant 2$ ). Strong interaction cross sections are approximately flat in $y$ in the central region. (Even though we are cutting out all events at $p_{\mathrm{T}} \leqslant 10$, we are at large enough energies so as not to alter the flat $y$ distribution.) The weak-interaction cross section has a fairly strong $y$ dependence. Fig. 5 shows a plot of $\mathrm{d} \widetilde{\sigma}^{\text {weak }} / \mathrm{d} y$ versus $y$ for $\overline{\mathrm{p}} \mathrm{p} \rightarrow \rho^{+}+\mathrm{X}$, where we have cut out the region $p_{\mathrm{T}} \leqslant 10$. Thus, in order to maintain the weakinteraction signal, we must make another cut preserving only a region about $y=0$. Let us choose to keep the region of $-1 \leqslant y \leqslant 1$. Then we can integrate over $y$ to obtain total cross sections:

$$
\begin{aligned}
& \widetilde{\sigma}_{\rho}^{\text {background }}=\left\{\begin{array}{lll}
4 \times 10^{-33} \mathrm{~cm}^{2}, & E_{\mathrm{b}}=100 \mathrm{GeV}, & p_{\mathrm{T}}>10 \mathrm{GeV} / c, \\
9 \times 10^{-33} \mathrm{~cm}^{2}, & E_{\mathrm{b}}=400 \mathrm{GeV}, & p_{\mathrm{T}}>10 \mathrm{GeV} / c,
\end{array}\right. \\
& \widetilde{\sigma}_{\rho}^{\text {weak }}=\left\{\begin{array}{lll}
1.5 \times 10^{-34} \mathrm{~cm}^{2}, & E_{\mathrm{b}}=100 \mathrm{GeV}, & p_{\mathrm{T}}>10 \mathrm{GeV} / c, \\
6.5 \times 10^{-34} \mathrm{~cm}^{2}, & E_{\mathrm{b}}=400 \mathrm{GeV}, & p_{\mathrm{T}}>10 \mathrm{GeV} / c .
\end{array}\right.
\end{aligned}
$$

Assume a luminosity of $L=10^{30} \mathrm{~cm}^{-2} \mathrm{sec}^{-1}$ (approximately what is expected for early $\bar{p}$ p colliding beams) $[9,10]$. Thus, the above numbers translate into:

$$
\begin{aligned}
& N_{\rho}^{\text {background }}= \begin{cases}346 \text { events } / \text { day, } & E_{\mathrm{b}}=100 \mathrm{GeV}, \\
778 \text { events } / \text { day }, & E_{\mathrm{b}}=400 \mathrm{GeV},\end{cases} \\
& N_{\rho}^{\text {weak }}= \begin{cases}13 \text { events } / \text { day }, & E_{\mathrm{b}}=100 \mathrm{GeV}, \\
56 \text { events } / \text { day, } & E_{\mathrm{b}}=400 \mathrm{GeV} .\end{cases}
\end{aligned}
$$

For comparison, we provide in table 2 the event rates expected at the CERN $\bar{p} p$ collider calculated from our cross sections.

How can we improve this situation? The numbers above suggest that the back- 


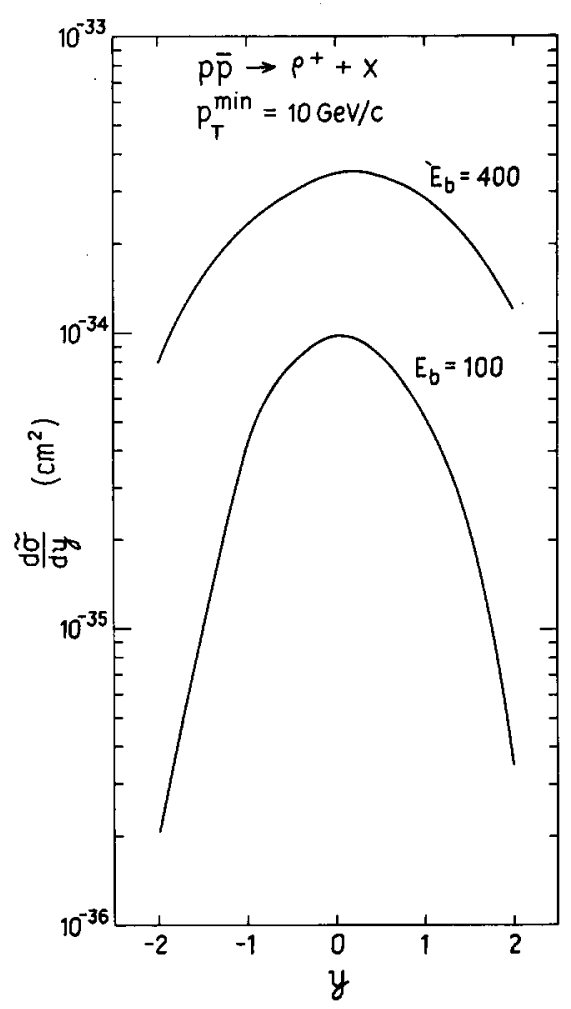

Fig. 5. Weak inclusive $\rho^{+}$production. The curves in fig. 4 have been integrated out in $p_{\mathrm{T}}$ starting from $p_{\mathrm{T}}=10 \mathrm{GeV} / c$.

Table 2

Expected event rates at the CERN p $\bar{p}$ collider

\begin{tabular}{|c|c|c|c|c|}
\hline \multirow[t]{2}{*}{ Event type } & \multicolumn{4}{|c|}{ Number of events/day at $E_{\mathbf{b}}=$} \\
\hline & 100 & 200 & 300 & 400 \\
\hline $\begin{array}{l}\mathrm{W}^{+} \rightarrow \text { all } \\
\mathrm{W}^{+} \rightarrow \mu^{+} \nu\end{array}$ & $\begin{array}{r}60 \\
5\end{array}$ & $\begin{array}{r}175 \\
14\end{array}$ & $\begin{array}{r}260 \\
21\end{array}$ & $\begin{array}{r}345 \\
28\end{array}$ \\
\hline $\begin{array}{l}\mathbf{Z}^{0} \rightarrow \text { all } \\
\mathbf{Z}^{0} \rightarrow \mu^{+} \mu^{-}\end{array}$ & $\begin{array}{r}25 \\
1\end{array}$ & $\begin{array}{r}95 \\
3\end{array}$ & $\begin{array}{r}175 \\
5\end{array}$ & $\begin{array}{r}190 \\
6\end{array}$ \\
\hline
\end{tabular}

We assume a luminosity of $10^{30} \mathrm{~cm}^{-2} \sec ^{-1}$. We use the $W$ and $Z$ cross sections given in figs. 16 and 17 and assume the following branching ratios (BR) into lepton pairs: $B R\left(W^{+} \rightarrow \mu^{+} \nu\right)=0.08$, $\operatorname{BR}\left(Z^{0} \rightarrow \mu^{+} \mu^{-}\right)=0.03$. (We have used a six-lepton six-quark model here; see eq. (A.14).) $E_{\mathrm{b}}$ is the energy of each beam in $\mathrm{GeV}$. 


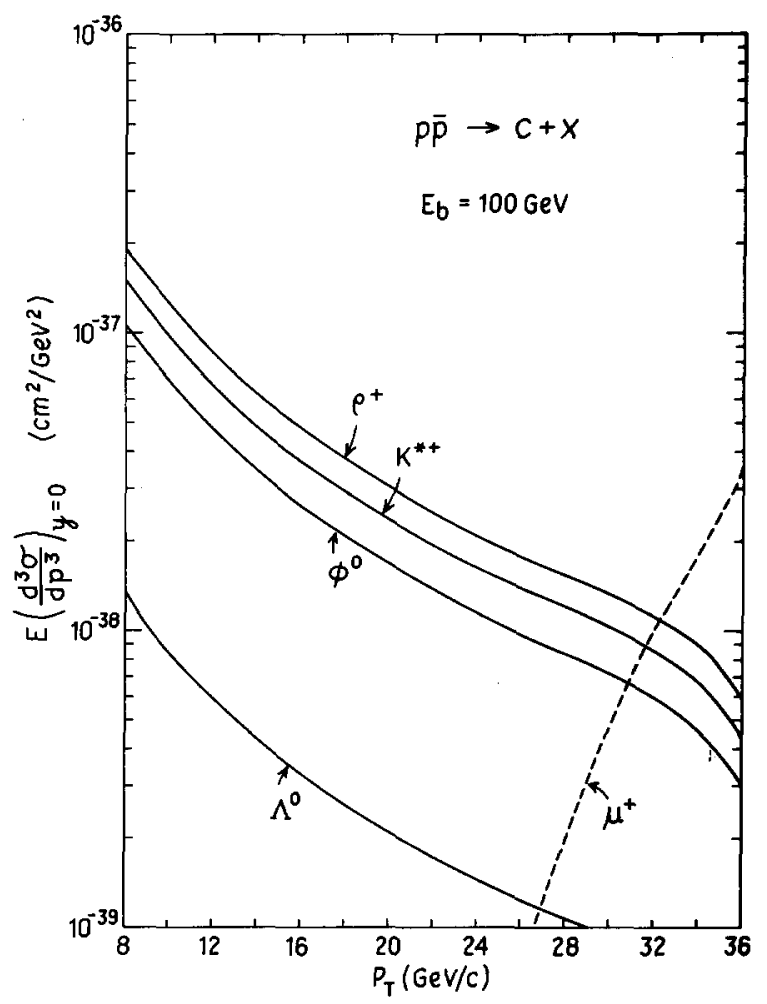

Fig. 6. Weak inclusive production at $E_{\mathrm{b}}=100 \mathrm{GeV}$. We compare inclusive production of $\rho^{+}, \mathrm{K}^{*+}$, $\varphi^{0}, \Lambda^{0}$, and $\mu^{+}$resulting from $W^{ \pm}$decay.

ground is still too large and would mask the weak effect. The signal-to-noise ratio could be enhanced in two ways:

(i) Cut out a larger $p_{\mathrm{T}}$ region, by choosing a larger value of $p_{\mathrm{T}}^{\min }$.

(ii) Look at the production of other resonances which might provide a larger weak signal as compared to the strong background. An example is $\vec{p} p \rightarrow \varphi^{0}+X$; this reaction has the advantage that the $\varphi^{0}$ is easy to detect (assuming good $\mathrm{K}$ identification). In addition, it is known [18] that at Fermilab the $\varphi / \pi$ ratio is $\approx 0.05$ for $p_{\mathrm{T}}>3 \mathrm{GeV} / c$. However, the $\mathrm{W}^{+}$boson decays into $\mathrm{c} \overline{\mathrm{s}}$ as easily as it does into $u \bar{d}$. So, we expect the $\varphi / \pi$ ratio at large $p_{\mathrm{T}}$ (produced by $\mathrm{W}^{+}$decay) to be much larger (perhaps as large as 0.5 ). Figs. 6 and 7 compare the cross sections for producing $\left(\right.$ via $\left.\mathrm{W}^{+}\right) \rho^{+}, \mathrm{K}^{*+}, \varphi^{0}$, and $\Lambda^{0}$.

Each of the methods above have the disadvantage of cutting the expected event rate further. Hence, at a luminosity of $10^{30} \mathrm{~cm}^{-2} \mathrm{sec}^{-1}$, our chances of seeing a weak signal by resonance production are rather poor. However, it is worth comparing these results with the single-lepton yield from a $\mathrm{W}^{+}$(see figs. 6, 7). In fact, the 


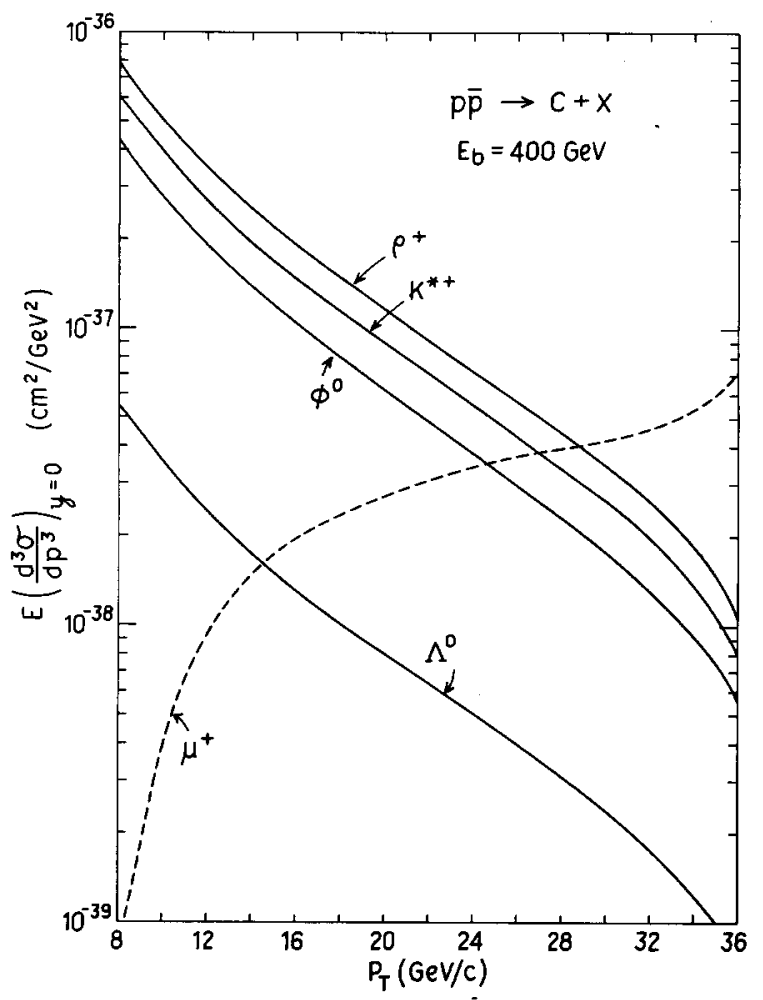

Fig. 7. Weak inclusive production at $E_{\mathrm{b}}=400 \mathrm{GeV}$. Same as fig. 6 .

background to the single-lepton signal is expected to be substantial. This suggests that at higher luminosities, resonance production could turn out to be a useful. signal of $\mathrm{W}$ production as well as a way to study $\mathrm{W}$ properties. If higher luminosities are not attainable at the proposed $\bar{p} p$ colliding machines, we may have to wait for Isabelle (pp colliding beams) for which a luminosity of $10^{33} \mathrm{~cm}^{-2} \mathrm{sec}^{-1}$ is anticipated.

Let us assume that a higher luminosity machine is available. We then can ask the question: what $p_{\mathrm{T}}$ cut is required so that the weak signal is about equal to the strong background? Referring to fig. 4 , let us choose $p_{\mathrm{T}}^{\min }=22 \mathrm{GeV} / c$ and repeat the previous calculations for $\mathrm{p} \overline{\mathrm{p}} \rightarrow \rho^{+}+\mathrm{X}$. We obtain:

$$
\begin{aligned}
& \left.\frac{\mathrm{d} \widetilde{\sigma}_{\rho}^{\text {background }}}{\mathrm{d} y}\right|_{y=0}=\left\{\begin{array}{lll}
2.5 \times 10^{-35} \mathrm{~cm}^{2}, & E_{\mathrm{b}}=100 \mathrm{GeV}, & p_{\mathrm{T}}>22 \mathrm{GeV} / c, \\
1.5 \times 10^{-34} \mathrm{~cm}^{2}, & E_{\mathrm{b}}=400 \mathrm{GeV}, & p_{\mathrm{T}}>22 \mathrm{GeV} / c,
\end{array}\right. \\
& \left.\frac{\mathrm{d} \widetilde{\sigma}_{\rho}^{\text {weak }}}{\mathrm{d} y}\right|_{y=0}=\left\{\begin{array}{lll}
3.5 \times 10^{-35} \mathrm{~cm}^{2}, & E_{\mathrm{b}}=100 \mathrm{GeV}, & p_{\mathrm{T}}>22 \mathrm{GeV} / c, \\
1.0 \times 10^{-34} \mathrm{~cm}^{2}, & E_{\mathrm{b}}=400 \mathrm{GeV}, & p_{\mathrm{T}}>22 \mathrm{GeV} / c .
\end{array}\right.
\end{aligned}
$$


Again, we integrate in the region of $-1 \leqslant y \leqslant 1$ and assume the background is flat in $y$. The weak signal has a similar $y$ dependence as before. We find:

$$
\begin{aligned}
& \widetilde{\sigma}_{\rho}^{\text {background }}=\left\{\begin{array}{lll}
5 \times 10^{-35} \mathrm{~cm}^{2}, & E_{\mathrm{b}}=100 \mathrm{GeV}, & p_{\mathrm{T}}>22 \mathrm{GeV} / c, \\
3 \times 10^{-34} \mathrm{~cm}^{2}, & E_{\mathrm{b}}=400 \mathrm{GeV}, & p_{\mathrm{T}}>22 \mathrm{GeV} / c,
\end{array}\right. \\
& \widetilde{\sigma}_{\rho}^{\text {weak }}=\left\{\begin{array}{lll}
5 \times 10^{-35} \mathrm{~cm}^{2}, & E_{\mathrm{b}}=100 \mathrm{GeV}, & p_{\mathrm{T}}>22 \mathrm{GeV} / c, \\
1.5 \times 10^{-34} \mathrm{~cm}^{2}, & E_{\mathrm{b}}=400 \mathrm{GeV}, & p_{\mathrm{T}}>22 \mathrm{GeV} / c .
\end{array}\right.
\end{aligned}
$$

Note that between $E_{\mathrm{b}}=100$ and $400 \mathrm{GeV}$ the background has increased more than the weak signal. We will return to this point later. If we choose a luminosity of $10^{30+n} \mathrm{~cm}^{-2} \mathrm{sec}^{-1}$, then we obtain event rates of:

$$
N_{\rho}^{\text {weak }}=\left\{\begin{array}{cc}
4 \times 10^{n} \text { events } / \text { day }, & E_{\mathrm{b}}=100 \mathrm{GeV}, \\
13 \times 10^{n} \text { events } / \text { day }, & E_{\mathrm{b}}=400 \mathrm{GeV},
\end{array}\right.
$$

with a signal-to-noise ratio of about $1: 1$. Thus, an increase in luminosity of the $p \bar{p}$ machines above $10^{30} \mathrm{~cm}^{-2} \mathrm{sec}^{-1}$ by a factor of 100 would make the detection of a weak signal feasible. If we repeat our calculations for $\mathrm{pp} \rightarrow \rho^{+}+\mathrm{X}$, we find (uncier identical conditions as above) that:

$$
N_{\rho}^{\text {weak }}= \begin{cases}0.15 \times 10^{n} \text { events } / \text { day }, & E_{\mathrm{b}}=100 \mathrm{GeV}, \\ 7 \times 10^{n} \text { events } / \text { day }, & E_{\mathrm{b}}=400 \mathrm{GeV} .\end{cases}
$$

Although the energy dependence here is different (favoring the higher energies over the lower ones), it is clear that at Isabelle with a luminosity of $10^{33} \mathrm{~cm}^{2} \mathrm{sec}^{-1}$ (i.e., $n=3$ in eq. (19)) our chances of seeing a signal are quite good.

We now turn to a detailed discussion of the weak signal itself. As our basic example, we will exhibit results for:

$$
\mathrm{p} \overline{\mathrm{p}} \rightarrow \mathrm{K}^{*}+\mathrm{X} \text {. }
$$

When we compute $\mathrm{d} \widetilde{\sigma} / \mathrm{d} y$, we must pick a point $p_{\mathrm{T}}^{\min }$ from which to integrate our inclusive cross sections. For the remainder of this paper, we have chosen $p_{\mathrm{T}}^{\min }=16$ $\mathrm{GeV} / c$, which is half way between the two values looked at previously. We hope that this will give the reader a fairly good idea of how our results change by choosing other values of $p_{\mathrm{T}}^{\min }$. In tables 3 and 4 , we present numerical results for $I_{1}, I_{2}, I_{3}$ and $I_{4}$ (see eq. 4) with $p_{\mathrm{T}}^{\min }=16 \mathrm{GeV} / c$. One can then compute $\mathrm{d} \widetilde{\sigma} / \mathrm{d} y$ for the production of various resonances (from $W$ decay) using table 1 .

We first examine the weak inclusive cross section predicted in the Weinberg-Salam model. In our model, $\mathrm{K}^{*+}$ would result from the decay of either a $\mathrm{W}^{+}$or a $\mathrm{Z}^{\mathbf{0}}$ boson. In fig. 8, we compare the results obtained at $y=0$ when we include or omit the $Z^{0}$. By leaving out the $Z^{0}$, we reduce the cross section by a factor of about 1.3. Similar results occur when we look away from $y=0$. The reason that the $\mathrm{Z}^{0}$ does not contribute more is directly attributable to the size of the Zq $\bar{q}$ couplings (see eq. (A.11) in the appendix). We conclude that our methods will not be able to identify the presence of the $Z^{0}$ until the signal due to the $\mathrm{W}^{+}$can be identified in detail. Thus 
Table 3

$\mathrm{d} \tilde{\sigma} / \mathrm{d} y$ for $\mathrm{p} \overline{\mathrm{p}} \rightarrow \mathrm{C}+\mathrm{X}$ via $\mathrm{W}$ exchange; $p_{\mathrm{T}}^{\min }=16 \mathrm{GeV} / \mathrm{c}$

(1). $E_{\mathrm{b}}=100 \mathrm{GeV}$

\begin{tabular}{rll}
\hline \multicolumn{1}{l}{$y$} & $I_{1}$ & $I_{2}$ \\
\hline-2.0 & $1.88(1)$ & $3.75(3)$ \\
-1.0 & $3.79(4)$ & $6.69(5)$ \\
-0.5 & $2.89(5)$ & $1.30(6)$ \\
0.0 & $9.34(5)$ & $1.10(6)$ \\
0.5 & $1.34(6)$ & $4.11(5)$ \\
1.0 & $8.51(5)$ & $6.50(4)$ \\
2.0 & $9.87(3)$ & $5.40(1)$ \\
\hline
\end{tabular}

(2). $E_{\mathrm{b}}=400 \mathrm{GeV}$

\begin{tabular}{rll}
\hline$y$ & $I_{1}$ & $I_{2}$ \\
\hline-2.0 & $1.29(5)$ & $1.11(6)$ \\
-1.0 & $1.56(6)$ & $2.91(6)$ \\
-0.5 & $2.55(6)$ & $3.34(6)$ \\
0.0 & $3.16(6)$ & $3.47(6)$ \\
0.5 & $3.42(6)$ & $3.16(6)$ \\
1.0 & $3.36(6)$ & $2.23(6)$ \\
2.0 & $1.71(6)$ & $2.56(5)$ \\
\hline
\end{tabular}

(3). $E_{\mathrm{b}}=1000 \mathrm{GeV}$

\begin{tabular}{|c|c|c|}
\hline$y$ & $I_{1}$ & $I_{2}$ \\
\hline-2.0 & $9.31(5)$ & $1.74(6)$ \\
\hline-1.0 & $2.03(6)$ & $2.23(6)$ \\
\hline-0.5 & $2.25(6)$ & $2.40(6)$ \\
\hline 0.0 & $2.37(6)$ & $2.57(6)$ \\
\hline 0.5 & $2.46(6)$ & $2.71(6)$ \\
\hline 1.0 & $2.53(6)$ & $2.71(6)$ \\
\hline 2.0 & $2.47(6)$ & $1.58(6)$ \\
\hline
\end{tabular}

The $I_{i}$ are defined by eq. (4). For p $\bar{p}$ inclusive scattering, via s-channel $\mathrm{W}$ exchange, $I_{3}(y)=I_{2}(-y)$ and $I_{4}(y)=I_{1}(-y)$ so we give only $I_{1}$ and $I_{2}$. Units are in $10^{-40} \mathrm{~cm}^{2}$; for example, $9.34(6)$ means $9.34 \times 10^{-34} \mathrm{~cm}^{2}$, etc.

for simplicity, for the rest of this section, we will omit the $Z^{0}$ contribution.

In order to identify a signal due to the $\mathrm{W}^{+}$, we must be able to prove that the $\mathrm{K}^{*+}$ was produced by a parity-violating interaction. Actually, this is a necessary but not a sufficient condition, since $\mathrm{K}^{*+}$ 's coming from weak decay of charmed particles would also be produced by a parity violating interaction. This background is likely to be negligible as can be seen from the following argument. Let us estimate the ratio 
Table 4

$\mathrm{d} \widetilde{\sigma} / \mathrm{d} y$ for $\mathrm{pp} \rightarrow \mathrm{C}+\mathrm{X}$ via $\mathrm{W}$ exchange; $p_{\mathrm{T}}^{\min }=16 \mathrm{GeV} / \mathrm{c}$

(1). $E_{\mathrm{b}}=100 \mathrm{GeV}$

\begin{tabular}{lllll}
\hline$y$ & $I_{1}$ & $I_{2}$ & $I_{3}$ & $I_{4}$ \\
\hline 0.0 & $1.75(4)$ & $4.91(4)$ & $6.93(3)$ & $1.61(4)$ \\
0.5 & $2.33(4)$ & $3.10(4)$ & $8.42(3)$ & $1.04(4)$ \\
1.0 & $2.23(4)$ & $7.37(3)$ & $7.01(3)$ & $2.64(3)$ \\
2.0 & $6.48(2)$ & $4.43(0)$ & $1.25(2)$ & $9.96(-1)$ \\
\hline
\end{tabular}

(2). $E_{\mathrm{b}}=400 \mathrm{GeV}$

\begin{tabular}{lllll}
\hline$y$ & $I_{1}$ & $I_{2}$ & $I_{3}$ & $I_{4}$ \\
\hline 0.0 & $8.03(5)$ & $1.83(6)$ & $4.74(5)$ & $9.91(5)$ \\
0.5 & $8.89(5)$ & $1.69(6)$ & $5.08(5)$ & $8.88(5)$ \\
1.0 & $1.06(6)$ & $1.22(6)$ & $5.64(5)$ & $5.93(5)$ \\
2.0 & $7.69(5)$ & $1.50(5)$ & $3.31(5)$ & $5.77(4)$ \\
\hline
\end{tabular}

(3). $E_{\mathrm{b}}=1000 \mathrm{GeV}$

\begin{tabular}{lllll}
\hline$y$ & $I_{1}$ & $I_{2}$ & $I_{3}$ & $I_{4}$ \\
\hline 0.0 & $1.30(6)$ & $2.14(6)$ & $8.65(5)$ & $1.31(6)$ \\
0.5 & $1.32(6)$ & $2.14(6)$ & $8.63(5)$ & $1.29(6)$ \\
1.0 & $1.40(6)$ & $2.07(6)$ & $8.63(5)$ & $1.20(6)$ \\
2.0 & $1.57(6)$ & $1.22(6)$ & $8.29(5)$ & $5.89(5)$
\end{tabular}

The $I_{i}$ are defined by eq. (4). For pp inclusive scattering, via s-channel $\mathrm{W}$ exchange, $I_{i}(-y)=I_{i}(y)$ so we omit negative values of $y$. Units are in $10^{-40} \mathrm{~cm}^{2}$; for example, $9.34(6)$ means $9.34 \times 10^{-34} \mathrm{~cm}^{2}$, etc.

of $\mathrm{D}^{+} / \pi^{+}$production at high $p_{\mathrm{T}}$. We know from experiments [19] that the integrated inclusive cross section for $\mathrm{pp} \rightarrow \mathrm{D}^{+}+\mathrm{X}$ is $\sigma \leqslant 50 \mu \mathrm{b}$. This would give an average multiplicity of $\left\langle n_{\mathrm{D}}\right\rangle=\sigma / \sigma_{\mathrm{pp}} \leqslant 10^{-3}$ (assuming a pp total cross section of $50 \mathrm{mb}$ at the energies we are interested in). At ISR energies [20], $\left\langle n_{\mathrm{K}}\right\rangle \approx 0.5$; thus, we obtain a $\mathrm{D}^{+} / \mathrm{K}^{+}$ratio of $\leqslant 2 \times 10^{-3}$. Let us assume this ratio will hold for large- $p_{\mathrm{T}}$ events as well; if we then use a $\pi / K$ ratio [21] of 2 for large- $p_{\mathrm{T}}$ events (note: such a ratio does not hold when low- $p_{\mathrm{T}}$ events are considered), we conclude that the $\mathrm{D}^{+} / \pi^{+}$ratio should be $\leqslant 10^{-3}$. To obtain the number of weakly produced $K^{*}$ 's coming from $D$ decay, we must multiply this number by a branching ratio; we take $B R\left(D \rightarrow K^{*}+\right.$ anything) $\leqslant 0.2$. Hence, we find that $K$ "s produced from charm particles would be found at a level of $\$ 2 \times 10^{-4}$ below the "strong" inclusive $\pi$ background shown in fig. 3 . We can safely neglect this possible source of contamination.

We can thus be sure that an observable parity violation could be attributed to-a 


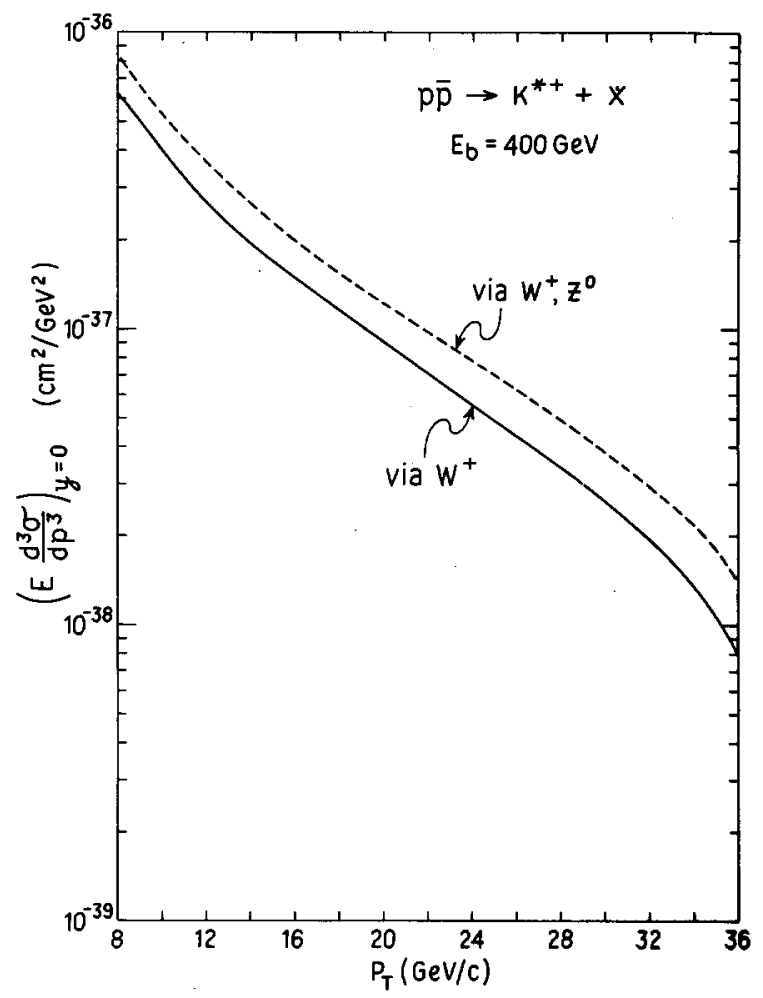

Fig. 8. Comparison of $\mathrm{W}^{+}$and $\mathrm{Z}^{0}$ production. The effect of adding in $\mathrm{Z}^{0}$ production to the inclusive production of $\mathrm{K}^{*+}$ is exhibited.

weak-interaction effect. In order to identify the parity violation experimentally, one must measure the decay density matrix elements of the $\mathrm{K}^{*}$. The relevant formalism is discussed in subsect. A.7 of the appendix. We can write:

$$
\rho_{\lambda \lambda} \frac{\mathrm{d} \sigma}{\mathrm{d} y}\left(\mathrm{p} \overline{\mathrm{p}} \rightarrow \mathrm{K}^{*}+\mathrm{X}\right)=\frac{\mathrm{d} \sigma}{\mathrm{d} y}\left(\mathrm{p} \overline{\mathrm{p}} \rightarrow \mathrm{K}^{*}(\lambda)+\mathrm{X}\right) .
$$

That is, the diagonal elements of the density matrix elements allow us to obtain the cross section for the production of $\mathrm{K}^{*}$ in a definite helicity state. This cross section is easily calculated in our model; details are given in subsect. A.5 of the appendix. Note that the off-diagonal elements of the density matrix involve the interference of amplitudes for producing $\mathrm{K}^{*}$ 's of different helicities. A simple parton model which just adds up quark-quark scatterings incoherently cannot predict such quantities. Therefore, the only signal of parity violation which our model can reliably 
predict at present is

$$
\alpha \equiv \frac{\rho_{11}-\rho_{-1-1}}{\rho_{11}+\rho_{-1-1}} .
$$

Parity conservation would imply $\alpha=0$; a non-zero $\alpha$ would indicate parity violation in the production of the $\mathrm{K}^{*}$. Unfortunately, as discussed in subsect. A.7 of the appendix, for systems which decay strongly, $\alpha$ cannot be measured with unpolarized beams. The reason for this is that the combination $\left(\rho_{11}-\rho_{-1-1}\right)$ does not appear in the expression for the strong decay angular distribution of the $\mathrm{K}^{*}$. Experimentally, one can measure parity violation by showing that $\operatorname{Im} \rho_{10} \neq-\operatorname{Im} \rho_{-10}$ or $\operatorname{Im} \rho_{1-1} \neq 0$. However, as discussed above, our model is unable to reliably calculate these quantities. Nevertheless, we expect the model to give a reasonable estimate of the amount of parity-violating production. For $\Lambda$ 's (see appendix) the asymmetry can be measured with unpolarized beams.

There are three possible approaches without expanding the present model. First, we can regard $\alpha$ as the "typical" size for a parity-violating parameter and hope that the experimentally observable parity-violating signals will also be of the same size as $\alpha$. To do this ignores any interference between strong and weak amplitudes. However, detecting parity violating production of $\mathrm{K}^{*}, \varphi, \rho, \Delta$, etc. via analysis of their strong decays means one is seeing an interference of strong and weak production. Assuming experiments are done in a region where weak production and background are comparable in size, and assuming that weak and strong amplitudes will not generally be in phase, we expect observable effects to be present. All the numbers we calculate and present will be for observables which are non-zero from purely weak effects, and go as weak quantities squared. Actual experimental observables could be weakstrong interferences and could be significantly larger than the quantities we present. As QCD calculations are better understood, it should be possible to estimate all the effects of interest, perhaps by the time experiments are feasible.

Second, we can search for a reaction where a parameter like $\alpha$ can be measured directly. It turns out that one needs to produce a hadron which has a parity-violating decay (such as $\Lambda^{0}$ ). We will discuss this case at the end of this section.

Third, we can consider polarized beams. In this case, there is no need to measure decay distributions since parity violation can be detected by measuring total cross sections. We discuss this in sect. 3. Our general approach in the following will be to assume that the parity-violating effects which we cannot reliably calculate are comparable in size to those which can be calculated.

We now return to the discussion of reaction (20). Let us assume that a parameter like $\alpha$ is observed and parity violation can be experimentally measured as a function of beam energy $E_{\mathrm{b}}$ (and other kinematic variables). We assume that experimental cuts are made to enhance the signal over background as previously discussed. It is crucial to make sure that the parity violation is real and not an artifact of the cuts that were made. For example, one should check that any signal present goes away when the cuts are slowly released (thus increasing the parity-conserving background). 


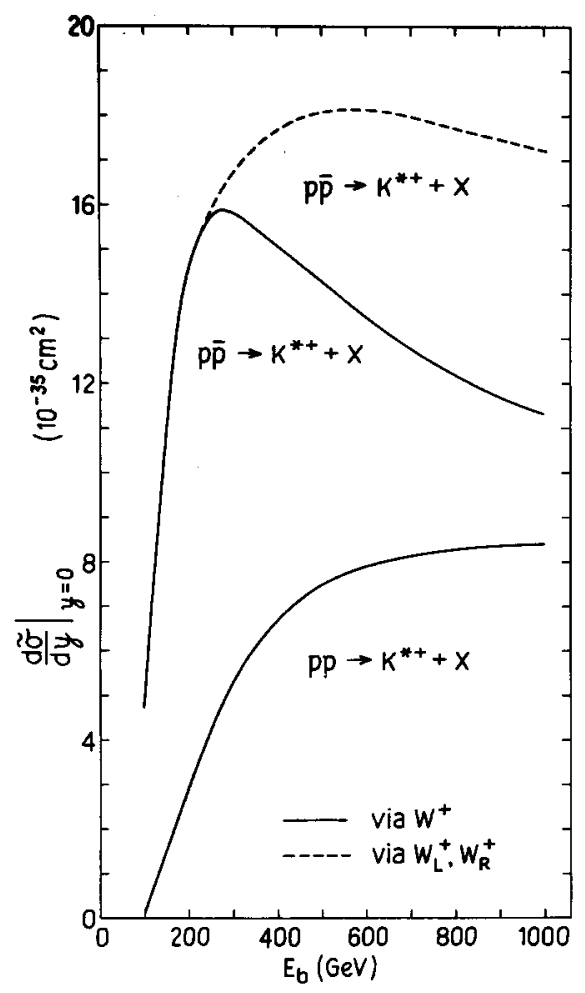

Fig. 9. Weak inclusive production of $\mathrm{K}^{*+}$ versus energy. Some gauge theories predict charged vector bosons $\left(\mathrm{W}_{R}\right)$ which couple to fermions as pure $V+A$. We have assumed that $m_{W_{R}}=250$ $\mathrm{GeV}$ as compared to the standard W-boson $\left(\mathrm{W}_{\mathrm{L}}\right)$ which has $m_{\mathrm{W}_{\mathrm{L}}}=75 \mathrm{GeV}$.

It is also very important to insure that the experimental cuts do not introduce an artificial energy dependence into the signal. In general, the energy dependence of the weak signal will yield information about the properties of the weak-interaction theory. For example, as shown in fig. 9 , in the standard theory the cross section for the reaction (20) peaks around $E_{\mathrm{b}}=300 \mathrm{GeV}$ and then slowly decreases. Note that this conclusion is valid only for $\bar{p} \mathrm{p}$ collisions and does not hold for pp collisions. This effect can be traced to the fact that at these energies, the dominant contribution to weak p p scattering is the scattering of two valence quarks. The peak in the $\mathrm{p} \overline{\mathbf{p}}$ cross section corresponds to the peak which occur in the valence quark distributions $x q(x)$ at $x \sim 0.2$. For pp scattering, which requires the interaction of valence and sea quarks, such a peak does not occur at the energies we are considering.

The above behavior can be different if we consider a more complicated gauge group. Consider an $\mathrm{SU}(2)_{\mathrm{L}} \otimes \mathrm{SU}(2)_{\mathrm{R}} \otimes \mathrm{U}(1)$ model which contains six massive gauge bosons: $W_{L}^{ \pm}, W_{R}^{ \pm}, Z_{A}^{0}$, and $Z_{\mathrm{V}}^{0}$. For the present discussion, we will neglect 


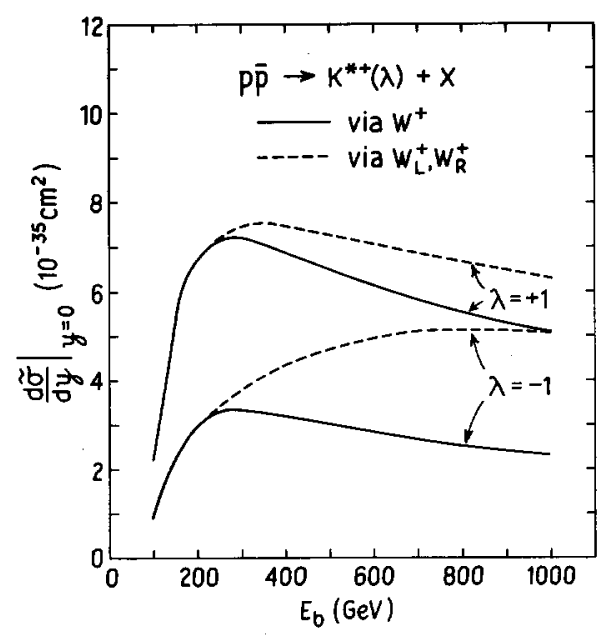

Fig. 10. Weak inclusive production of polarized $K^{*+}$ versus energy. Note that $\sigma\left(\mathrm{p} \overline{\mathrm{p}} \rightarrow \mathrm{K}^{*+}(\lambda)+\mathrm{X}\right)$ $\rho_{\lambda \lambda} \sigma\left(\mathrm{p} \overline{\mathrm{p}} \rightarrow \mathrm{K}^{*+}+\mathrm{X}\right)$ where $\rho$ is the density matrix (see appendix, A.7). See caption to fig. 9.

the neutral vector bosons. The $\mathrm{W}_{\mathrm{L}}^{ \pm}$are just the $\mathrm{W}^{ \pm}$of the Weinberg-Salam model. We consider the model of Mohapatra and Sidhu [22] where fermions appear in both left-handed and right-handed doublets; e.g.,

$$
\left(\begin{array}{l}
u \\
d
\end{array}\right)_{L}, \quad\left(\begin{array}{l}
u \\
d
\end{array}\right)_{R} \text {, etc. }
$$

The $W_{L}\left(W_{R}\right)$ mediates transitions from $u_{L}\left(u_{R}\right)$ to $d_{L}\left(d_{R}\right)$. Since right-handed currents are absent in low-energy weak-interaction phenomenology, we find that necessarily $m_{\mathrm{W}_{\mathrm{R}}} \gg m_{\mathrm{W}_{\mathrm{L}}}$. Actually, the lower bound for $m_{\mathrm{W}_{\mathrm{R}}} / m_{\mathrm{W}_{\mathrm{L}}}$ required by the low-energy data is not very stringent; Beg et al. [23] find $m_{\mathrm{W}_{\mathrm{R}}} / m_{\mathrm{W}_{\mathrm{L}}} \geq 2.8$. Let us take as an example $m_{\mathrm{W}_{\mathrm{L}}}=75 \mathrm{GeV}$ and $m_{\mathrm{W}_{\mathrm{R}}}=250 \mathrm{GeV}$. Then, the peak in the cross section for reaction $(20)$ is shifted to larger energies as can be seen in fig. 9 . However, this $\mathrm{SU}(2)_{\mathrm{L}} \otimes \mathrm{SU}(2)_{\mathrm{R}} \otimes \mathrm{U}(1)$ model [22] has the feature that the weak interactions become parity conserving at energies much larger than $m_{W_{R}}$, which is shown very nicely in figs. 10 and 11 . Thus, by detecting the weak production of hadron resonances it may be possible to distinguish among different weak-interaction theories.

Let us examine more carefully the cross sections for the production of $\mathrm{K}^{*+}$ 's of definite helicity, depicted in fig. 12. Notice that in the region around $y=0$, the helicity $+1 \mathrm{~K}^{*+}$ 's are about twice as copious as helicity $-1 \mathrm{~K}^{*+1} \mathrm{~s}$. The reason for this is that the $\mathrm{W}^{+}$decays into left-handed quarks and right-handed antiquarks. The helicity $+1 \mathrm{~K}^{*+}$ 's are the result of right-handed s-quarks which pick up a $\overline{\mathrm{u}}$-quark from the vacuum, while the helicity $-1 \mathrm{~K}^{*+}$ 's are the result of left-handed u-quarks 


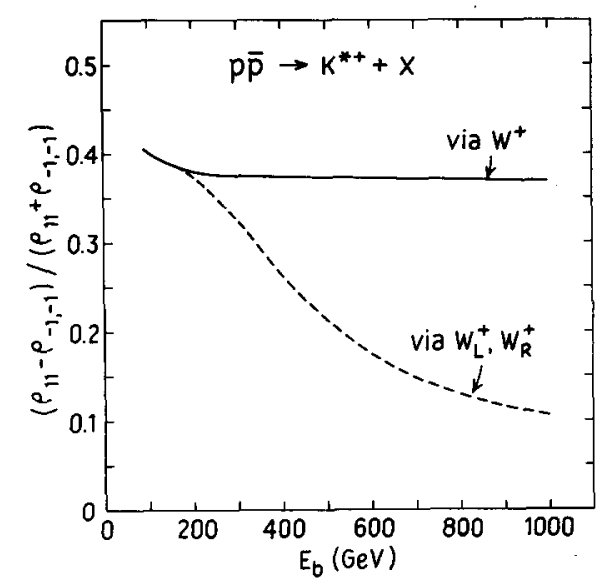

Fig. 11. Parity violation in inclusive $K^{*+}$ production. Parity conservation implies $\rho_{11}=\rho_{-1,-1}$. Hence $\left(\rho_{11}-\rho_{-1,-1}\right) /\left(\rho_{11}+\rho_{-1,-1}\right)$ is one measure of parity violation in the production of the $\mathrm{K}^{*+}$. No "strong" background is included here (however, see fig. 22). See captions to figs. 9, 10 and discussion in text.

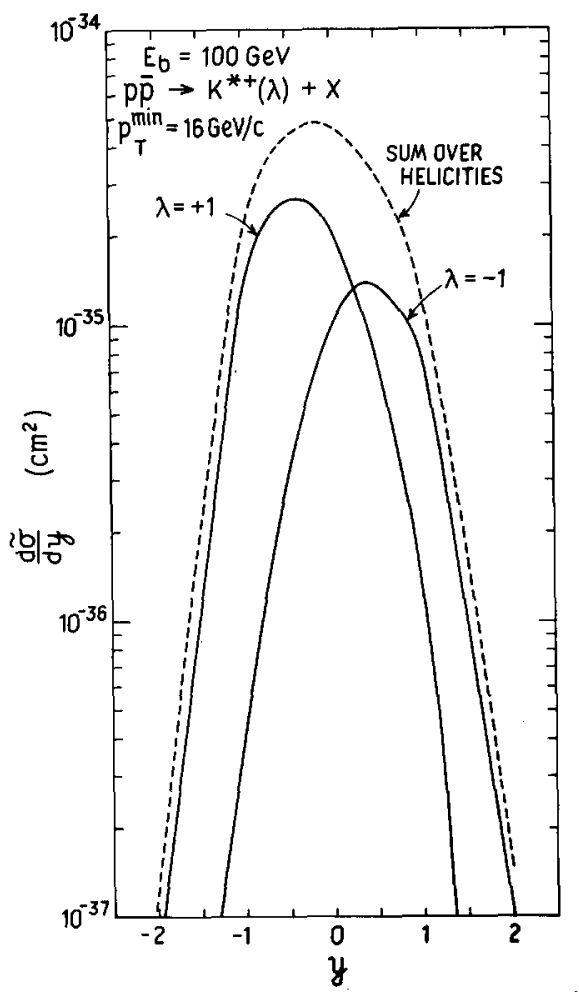

Fig. 12. Weak inclusive production of polarized $\mathrm{K}^{*+}$. 


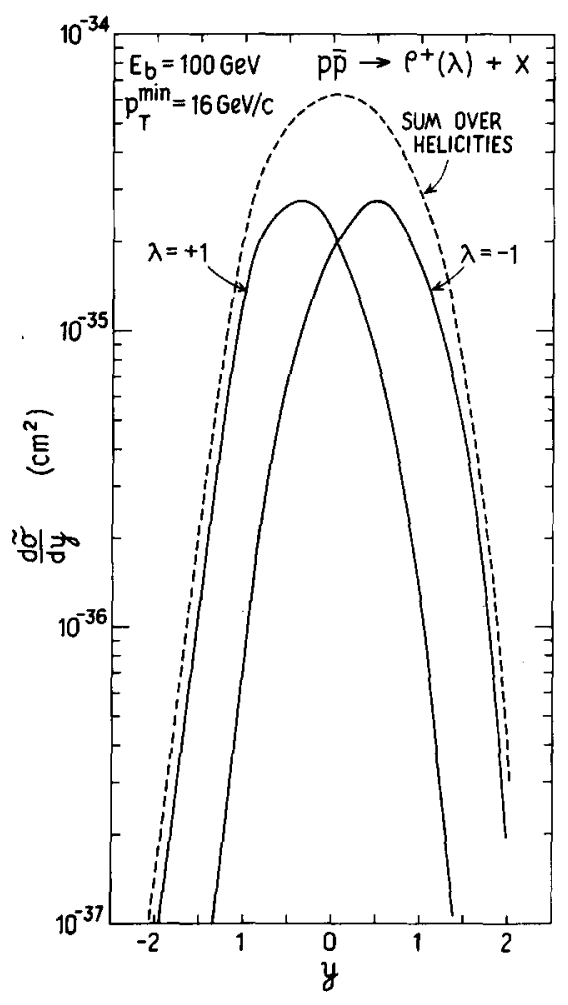

Fig. 13. Weak inclusive production of polarized $\rho^{+}$.

which pick up an $\bar{s}$-quark from the vacuum. But we have assumed that it is twice as easy to pick up a u-quark from the vacuum as compared to an s-quark [14]. This is to be compared with the weak inclusive production of $\rho$-mesons in fig. 13. Even though the cross sections for producing helicity $\pm 1 \rho$ 's differ, one must make careful cuts in $y$ to observe the effect.

As in the case of the $\rho$, the $\varphi$ meson poses similar problems (see fig. 14). However, we would like to emphasize a point already mentioned with regards to the $\varphi^{0}$ meson. A dramatic increase of the $\varphi / \pi$ ratio above $W$ threshold might be strong enough evidence to suggest the presence of weak interactions without identifying the parity violation. Comparing figs. 13,14 we estimate a $\varphi / \rho$ ratio of 0.5 which could be a factor of 10 above the ratio occurring in strong inclusive production. Furthermore, with the strong background for the $\varphi$ lower than for other hadrons, it should be easier to identify parity violation by measuring the $\varphi$ decay angle distribution.

We conclude this section by discussing inclusive baryon production. We admit that the cross sections are not optimistic (see figs. 6,7). But, if experiments become 


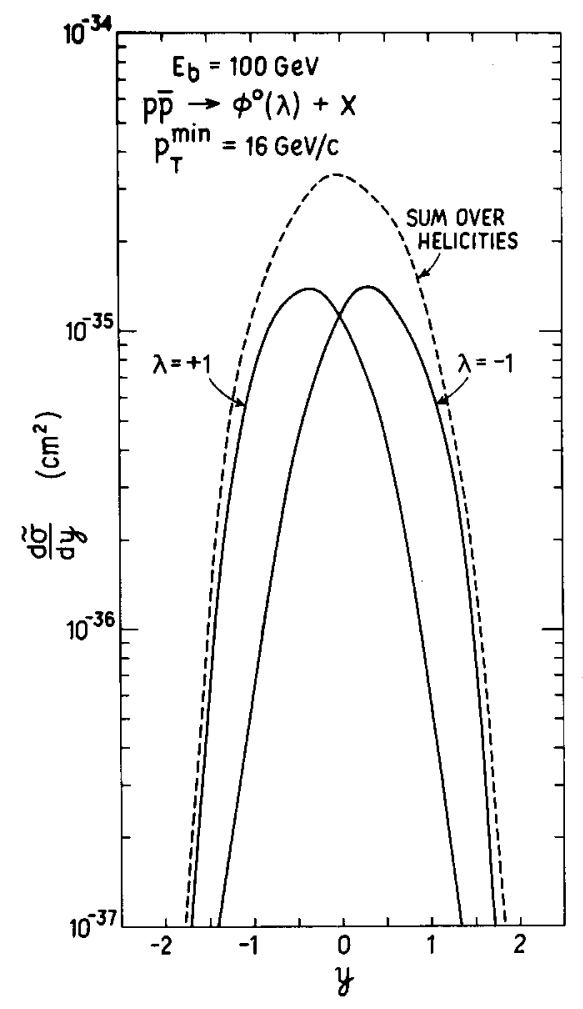

Fig. 14. Weak inclusive production of polarized $\varphi^{0}$.

sensitive enough, $\Lambda$ production would be an ideal laboratory in which to study the weak interactions at very high energies. As discussed in subsect. A.7 of the appendix, because the $\Lambda$ decays weakly, by measuring its decay angle distribution, one can measure the parity-violating term $\rho_{1 / 2}, 1 / 2-\rho_{-1 / 2},-1 / 2$ directly. This corresponds to the difference in cross sections for the production of helicity $\pm \frac{1}{2} \Lambda^{0}$ 's; see fig. 15 . For baryons such as $\Delta$ with parity conserving decays, the parity violating terms $\rho_{1 / 2,1 / 2} \cdot \rho_{-1 / 2,-1 / 2}$ and $\rho_{3 / 2,3 / 2}-\rho_{-3 / 2,-3 / 2}$ cannot be measured directly. We expect fairly large differences in the production of the various helicity states of the $\Delta$. For example, in our simple model, left-handed quarks will never produce a helicity $+\frac{3}{2} \Delta$ but can produce a helicity $-\frac{3}{2} \Delta$. We would guess, however, that $\Delta$ production would be a factor of ten below $\rho$ production (similar to the $\mathrm{p} / \pi$ ratio [21] at large $p_{\mathrm{T}}$ ). Thus, as in the case of $\Lambda$ production, very sensitive experiments would be required to identify a signal. 


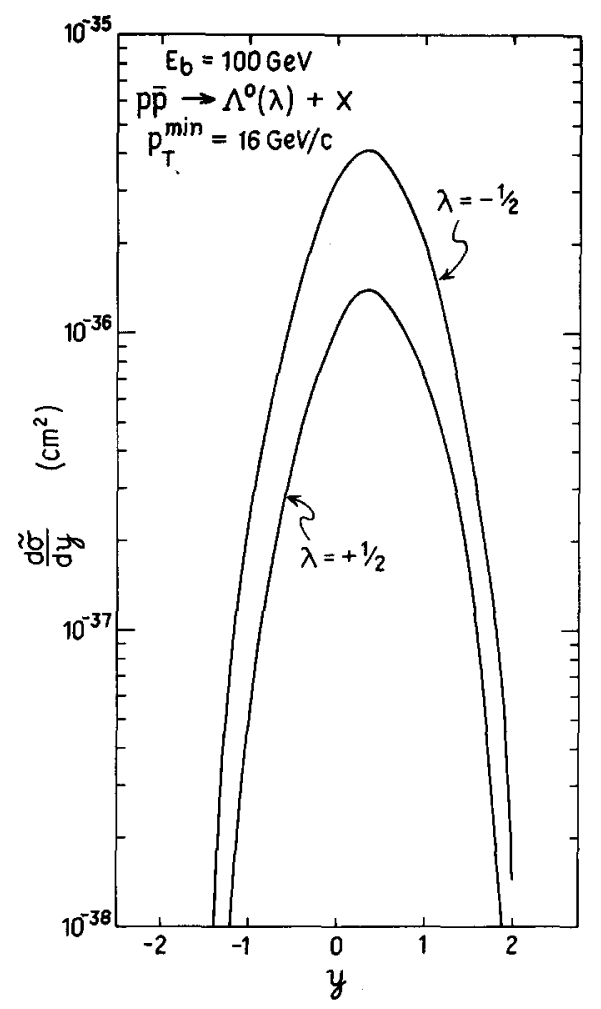

Fig. 15. Weak inclusive production of polarized $\Lambda^{0}$. Note the change in scale as compared with figs. $12-14$.

\section{Detection of weak effects via polarized beams}

Recently, there have been discussions [24] on the feasibility of polarized beams at very large energies. This would certainly add an important method for the detection of weak interactions at high energies. A number of authors $[5,25]$ have calculated the total $\mathrm{pp}$ cross sections with polarized beams taking into account the effects of weak interactions. In this section, we present results in the energy range above $W$ threshold. Details of the calculation are given in subsect. A.6 of the appendix.

First we consider the cross sections:

$$
\begin{aligned}
& \mathrm{p} \overline{\mathrm{p}} \rightarrow \mathrm{W}^{ \pm}, \mathrm{Z}^{0}+\mathrm{X}, \\
& \mathrm{p} \mathrm{p} \rightarrow \mathrm{W}^{ \pm}, \mathrm{Z}^{0}+\mathrm{X},
\end{aligned}
$$

where the beams are assumed to be longitudinally polarized (i.e., in definite helicity states). The general formula for the cross section is given by eq. (A.37) of the appen- 


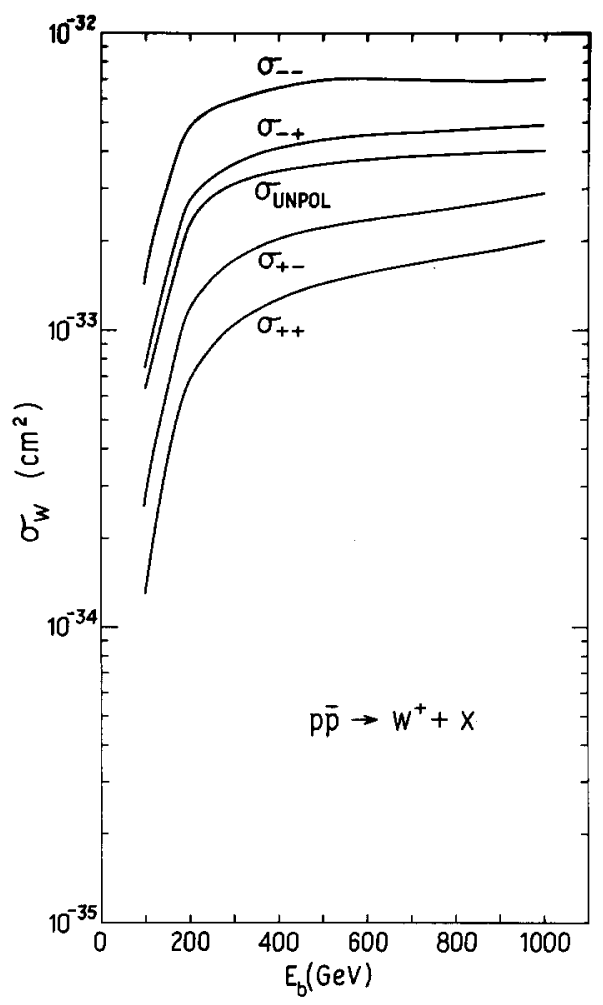

Fig. 16. Drell-Yan prediction for $\mathrm{p} \overline{\mathrm{p}} \rightarrow \mathrm{W}^{+}+\mathrm{X}$ with polarized beams. The notation here is $\sigma_{\lambda \lambda^{\prime}}$ where $\frac{1}{2} \lambda$ is the helicity of the proton and $\frac{1}{2} \lambda^{\prime}$ is the helicity of the antiproton.

dix; this is just a generalization of the standard Drell-Yan [26] formula. The only new ingredient required in this case is a set of "polarized" quark distribution functions (see subsect. A.3 of the appendix). We have calculated the cross sections $\sigma_{\lambda \lambda^{\prime}}$ for reactions (23); $\frac{1}{2} \lambda$ is the helicity of the proton in reaction (23a). The unpolarized cross section is obtained by averaging over the four possible $\sigma_{\lambda \lambda^{\prime}}$. These results agree with the results obtained by other authors $[1,2]$; small differences can be attributed to different quark distribution functions or a different choice of $\sin \theta_{\mathrm{w}}$. We present our results in figs. 16-20. To observe parity violation, one can measure total pp and $\mathrm{p} \overline{\mathrm{p}}$ cross sections where one or both beams are in definite helicity states. If we denote the total cross sections measured by $\left(\sigma_{\mathrm{T}}\right)_{\lambda \lambda^{\prime}}$, then:

$$
\left(\sigma_{\mathrm{T}}\right)_{\lambda \lambda^{\prime}}-\left(\sigma_{\mathrm{T}}\right)_{-\lambda,-\lambda^{\prime}}=\sigma_{\lambda \lambda^{\prime}}-\sigma_{-\lambda,-\lambda^{\prime}},
$$

where $\sigma_{\lambda \lambda^{\prime}}$ is the sum of all cross sections for parity-violating processes. But, the parity-violating processes are just those processes due to weak-interaction effects, i.e., the reactions (23) for any appropriate decay of W, Z. Hence, in order to identify 


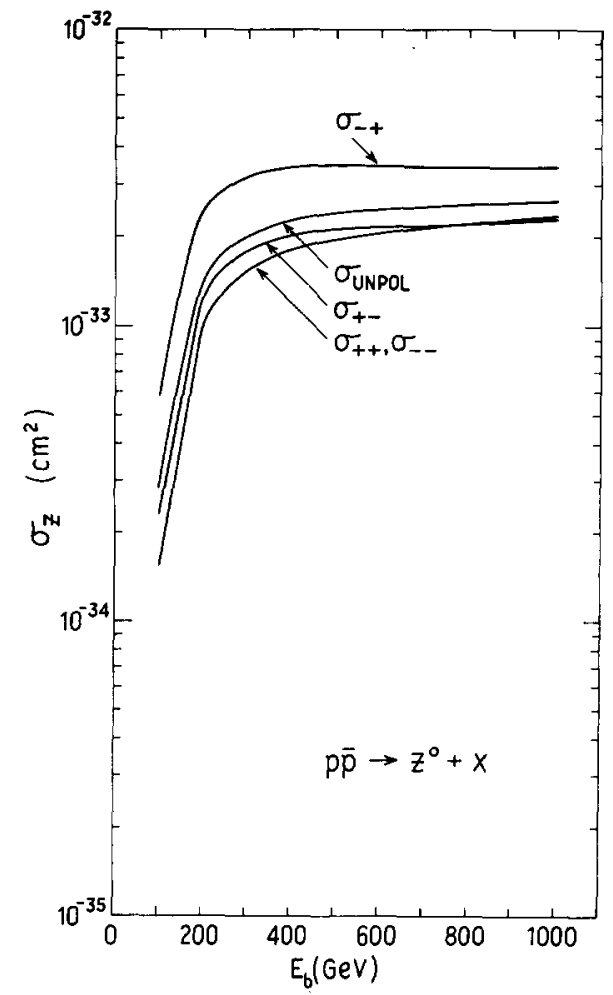

Fig. 17. Drell-Yan prediction for $\mathrm{p} \overline{\mathrm{p}} \rightarrow \mathrm{Z}^{0}+\mathrm{X}$ with polarized beams. See caption to fig. 16 .

parity violation one may identify the eixstence of any of the following three asymmetries:

$$
\begin{aligned}
& \alpha_{1}=\frac{\sigma_{++}-\sigma_{-二}}{\sigma_{\mathrm{T}}}, \\
& \alpha_{2}=\frac{\sigma_{+-}-\sigma_{-+}}{\sigma_{\mathrm{T}}}, \\
& \alpha_{3}=\frac{\sigma_{+}-\sigma_{-}}{\sigma_{\mathrm{T}}},
\end{aligned}
$$

where $\sigma_{\lambda}=\frac{1}{2} \Sigma_{\lambda^{\prime}} \sigma_{\lambda \lambda^{\prime}}$ and $\sigma_{\mathrm{T}}$ is the total pp (or p p) cross section. Note that $\alpha_{3}=\alpha_{1}$ $+\alpha_{2}$. Since $\sigma_{\mathrm{T}} \approx 50 \mathrm{mb}$, we see that in general the $\alpha_{i}$ are of order $10^{-8}$. Clearly, very precise measurement will be required to measure this effect.

It is worth remarking that $\sigma_{++}-\sigma_{+-} \neq 0$ does not imply parity violation. Indeed, 


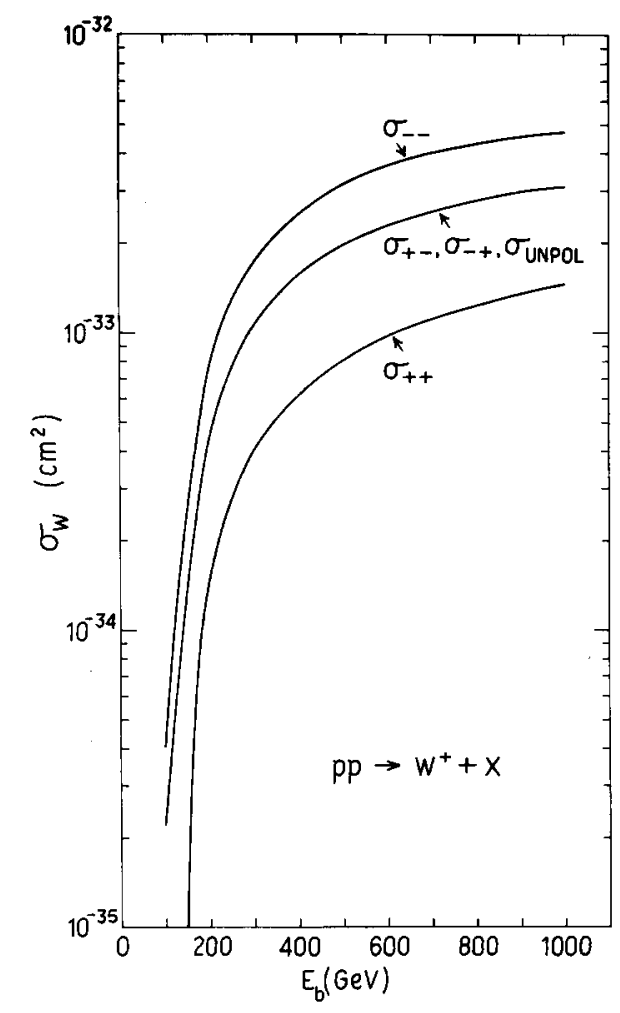

Fig. 18. Drell-Yan prediction for $\mathrm{pp} \rightarrow \mathrm{W}^{+}+\mathrm{X}$ with polarized beams.

this difference is about $1 \mathrm{mb}$ [27] at $6 \mathrm{GeV} / c$, presumably due to $A_{1}$ trajectory exchange [28]. Extrapolating with $p_{1 \mathrm{ab}}^{-1}$, an asymmetry of a few parts in $10^{-7}$ is expected from hadronic effects at $E_{\mathrm{b}}=400 \mathrm{GeV} / c$. Of course, weak effects can contribute as well.

An approach to increase the size of the signal would be to reduce the size of the denominators in eq. (25) by triggering on very high- $p_{T}$ events. All of our discussion of background in sect. 4 applies here. One should measure the cross section for inclusive production of any positively charged hadron at large $p_{\mathrm{T}}$ (greater than some $p_{\mathrm{T}}^{\mathrm{min}}$ ) in the vicinity of $\theta_{\mathrm{cm}}=90^{\circ}$ (say, $\left.|y| \leqslant 1\right)$. To calculate the prediction of our model, we must insert the results of eq. (A.35) into eq. (A.10). Note that in this instance the sum over $i$ and $j$ refers to the sum over all initial quarks of specific helicity; i.e., we must use the "polarized" quark functions. The result then will be a generalization of eq. (2). We could calculate the inclusive cross section for a specific hadron. But since it doesn't matter which hadron we observe, let us sum over all outgoing positively charged hadrons. To do this correctly (assuming only W production), 

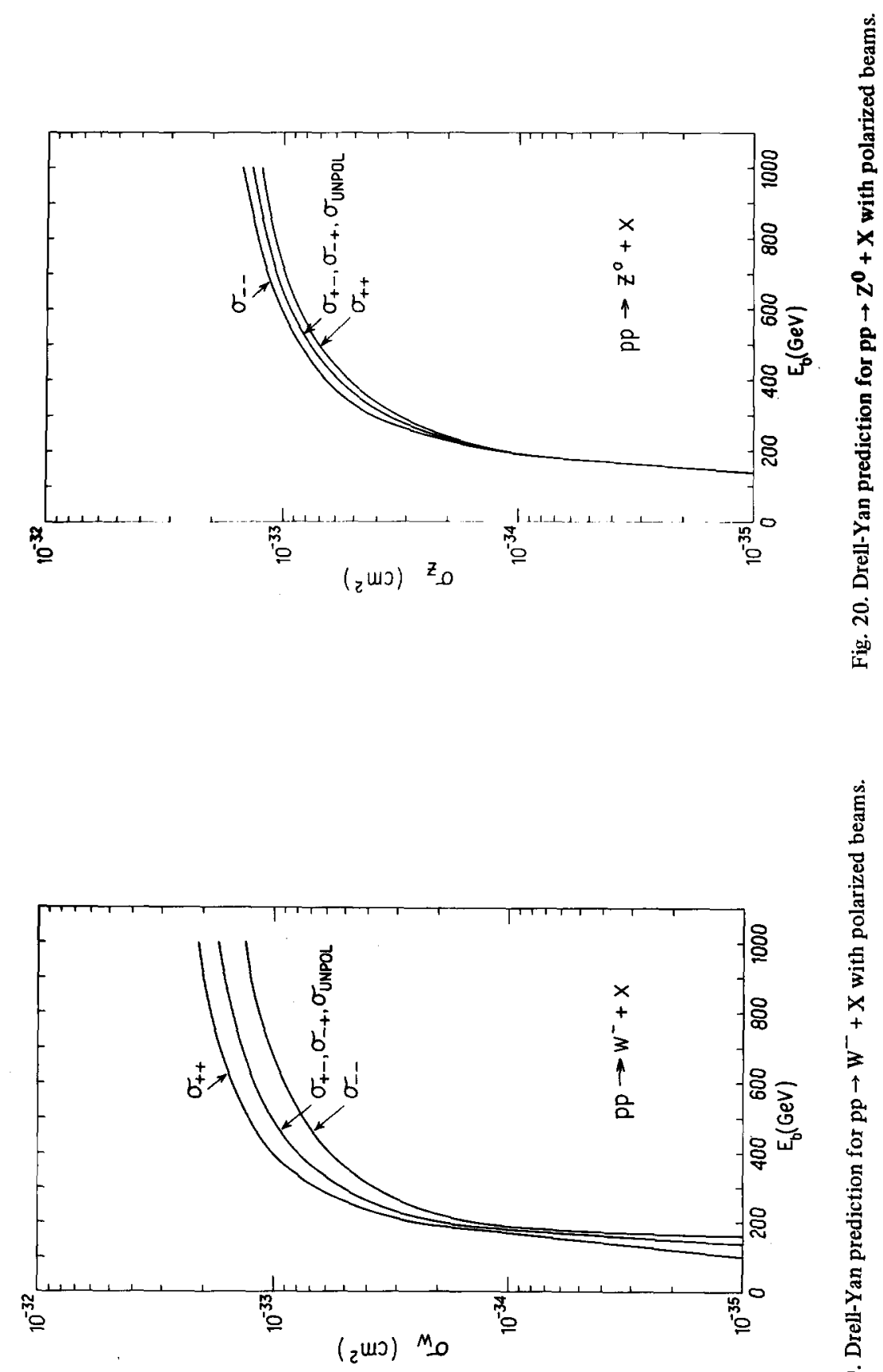

告 


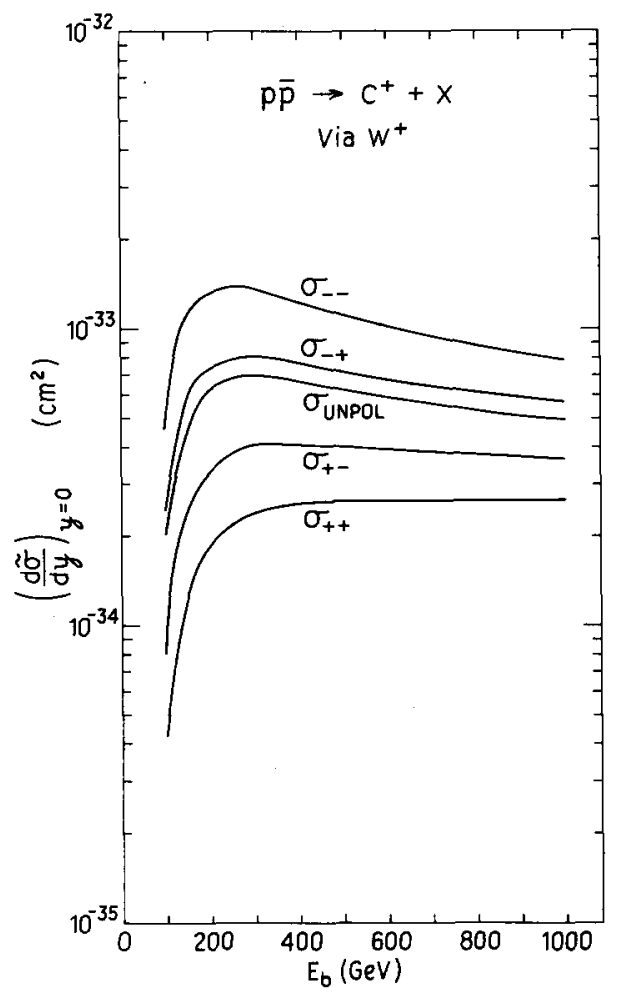

Fig. 21. Weak inclusive production of positively charged hadrons with polarized beams. See caption to fig. 16 .

we would have to add up expressions similar to the ones given in table 1 . We would also have to allow for the vector mesons to decay into $\pi$ 's and K's. Since we are only interested in a rough estimate here, we have simply calculated the inclusive cross section $\mathrm{p} \overline{\mathrm{p}} \rightarrow \rho^{+}+\mathrm{X}$ but used $K=1$ in eq. (5). The result is given in fig. 21 ; note the similarity to fig. 16 . If we now estimate typical values for the $\alpha_{i}$ of eq. (25), $\sigma_{\mathrm{T}}$ is replaced by the sum of the weak cross sections and background. Thus, typical values of $\alpha_{i}$ are of order $10^{-2}$ if we assume backgrounds of order $10^{-32} \mathrm{~cm}^{2}$. However, we must consider the fact that the event rate for large $p_{\mathrm{T}}$ events will be very small. The time required to see enough events to obtain a statistically significant asymmetry of $10^{-2}$ will depend directly on the luminosity of the colliding beam machines. Careful considerations of the luminosity and the "strong" background will reveal whether this method will be superior to measuring total cross sections. 


\section{Detection of other weak effects at high energies}

Let us assume that the intermediate vector bosons will be discovered at the next generation colliding beam machines. Once the properties of the weak vector bosons are studied and well-understood, it becomes possible to investigate other weak-interaction phenomena. The obvious question to ask is: will large weak-interaction effects (not attributable to the production of weak vector bosons) be observable at very high energies? This is both a theoretical and experimental question. In a recent paper [29], we have discussed the likelihood of finding such large weak effects in the context of the gauge theories. We found that in conventional gauge theories, such effects are suppressed; in theories with more than one Higgs multiplet, observable effects may be possible. We will take a phenomenological view here. Suppose that large unconventional weak effects do exist: could they be distinguished from conventional $\mathrm{W}$ and $\mathrm{Z}$ production?

Since we don't know a priori what properties these unconventional effects might have, such a question is difficult to answer. A number of authors [30] have noted that the weak interactions could exhibit strong-interaction properties at very high energies. A consequence of this could be the existence of bound states of the weak vector bosons and the Higgs bosons. Furthermore, the lowest-mass bound state might be less massive than the $\mathrm{W}$ boson. It would be interesting to imagine how one could observe experimentally whether such effects existed.

In order to see how a non-standard weak-interaction effect might differ from the results we obtained in sect. 2 , we chose the following model. Suppose the quarkquark cross section resembles diffractive elastic scattering; that is, we write:

$$
\frac{\mathrm{d} \sigma}{\mathrm{d} \hat{t}}=16 \pi B^{2} f^{2} \mathrm{e}^{B \hat{t}},
$$

where $B$ and $f$ are unknown parameters. (We have obtained this from an amplitude of the form $M=-i s \sigma_{\mathrm{T}} \mathrm{e}^{B t / 2}$; using the optical theorem, we see that $f \equiv \sigma / \sigma_{\mathrm{T}}$.)

Furthermore, let us assume that this non-standard weak interaction is $\mathrm{V}-\mathrm{A}$. Then, in the notation of subsect. A.5 of the appendix, we can write:

$$
F_{-+}\left(\hat{x}_{1}, \hat{x}_{2}\right)=16 \pi B^{2} f^{2} \hat{s} \mathrm{e}^{-B \hat{x} \hat{x}_{2}},
$$

and all other $F_{\lambda \lambda^{\prime}}$ are zero. We can now insert eq. (27) into eq. (A.10) of the appendix and derive results similar to those in sect. 2.

There is no reason to believe that results obtained from eq. (26) have anything to do with reality. But, it is amusing to observe how a non-standard weak-interaction effect might differ from the standard ones. We have chosen $f=0.25$ and $B=10^{-3}$ $\mathrm{GeV}^{-2}$ and have calculated $\rho_{11^{-} \rho_{-1,-1}}$ for the $\mathrm{K}^{*+}$ decay in $\mathrm{p} \overline{\mathrm{p}} \rightarrow \mathrm{K}^{*+}+\mathrm{X}$. We have included a strong interaction background (see subsect. 2.3) which rises slowly with energy, and we have cut out the kinematic region consisting of $p_{\mathrm{T}} \leqslant 16 \mathrm{GeV} / c$ and $|y| \geqslant 1$. In fig. 22 we compare the results of fig. 11 with the result obtained 


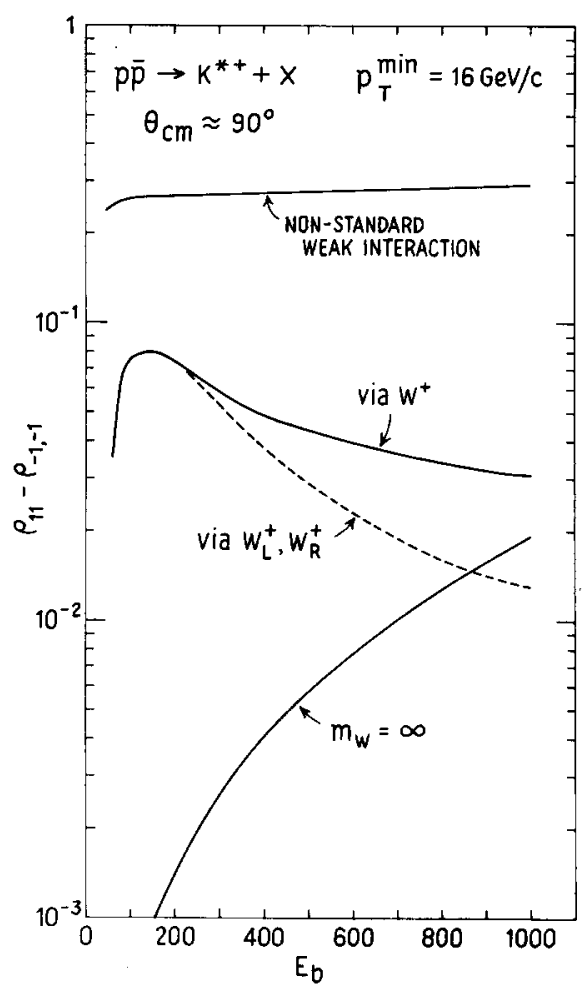

Fig. 22. $\rho_{11}-\rho_{-1,-1}$ for the $\mathrm{K}^{*+}$ decay in $\mathrm{p} \overline{\mathrm{p}} \rightarrow \mathrm{K}^{*+}+\mathrm{X}$. We have plotted results for four models: a four-fermion weak interaction model $\left(m_{\mathrm{W}}=\infty\right)$, the two models used in fig. 10 and a non-standard weak-interaction effect resulting from eq. (26) in the text. The strong-interaction background is included here. We have included the kinematic region of $p_{\mathrm{T}}>16 \mathrm{GeV} / \mathrm{c}$ and $|y| \leqslant 1$.

when we use eq. (26). For completeness, we have also included the case where we assume a four-fermion coupling of the quarks (i.e., the limit of $m_{\mathrm{W}} \rightarrow \infty$ ).

In the present example, we can see immediately that both the energy dependence and the magnitude of the non-standard weak interaction clearly differentiate it from the standard effects. It is also interesting to note that the four-fermion coupling leads to a negligible signal at lower energies, but the signal does increase steadily with energy.

The discovery of the intermediate vector bosons at the next generation colliding beam machines (assuming it occurs) will be one of the major events in high-energy physics in the 1980s. But the possibility of discovering or excluding unexpected phenomena at very high energies is even more exciting. To test the gauge theories it will be necessary to go beyond the discovery of W's and Z's. Presumably, nature will not choose an unconventional weak-interaction signal as dramatic as the one depicted in 
fig. 22. Nevertheless, it is important to search for these effects; their presence or absence will have a crucial effect on our understanding of weak interactions.

We appreciate helpful discussions with R. Cahn, M. Einhorn, S. Ellis, L. Lederman and B. Weeks.

\section{Appendix}

In this appendix, we collect all the formulas used in obtaining the graphs in this paper. Additional details are available in ref. [31].

\section{A. 1. Kinematics and the inclusive cross section}

We begin by defining the kinematic variables describing inclusive scattering $\mathrm{A}+\mathrm{B} \rightarrow \mathrm{C}+\mathrm{X}$. Let $p_{\mathrm{A}}, p_{\mathrm{B}}$, and $p_{\mathrm{C}}$ be the four-momenta of particles $\mathrm{A}, \mathrm{B}$, and $\mathrm{C}$ respectively. We define

$$
\begin{aligned}
& x_{1}=\frac{-u}{s}, \\
& x_{2}=\frac{-t}{s},
\end{aligned}
$$

where $s=-\left(p_{\mathrm{A}}+p_{\mathrm{B}}\right)^{2}, t=-\left(p_{\mathrm{A}}-p_{\mathrm{C}}\right)^{2}$, and $u=-\left(p_{\mathrm{B}}-p_{\mathrm{C}}\right)^{2}$. In the c.m.s., we define the positive $z$-axis to lie along the direction of particle A. Particle $C$ has energy $E$ in this frame and emerges at an angle $\theta_{\mathrm{cm}}$. In the high-energy limit (where we can neglect all masses), the transverse momentum $p_{\mathbf{T}}$ and the longitudinal momentum $p_{\|}$of particle $\mathrm{C}$ are given by

$$
\begin{aligned}
& p_{\mathrm{T}}=E \sin \theta_{\mathrm{cm}}, \\
& p_{\|}=E \cos \theta_{\mathrm{cm}} .
\end{aligned}
$$

The c.m. rapidity $y$ is defined as:

$$
y=\frac{1}{2} \ln \frac{E+p_{\|}}{E-p_{\|}} .
$$

In the limit where $p_{\mathrm{T}} \gg m_{\mathrm{C}}, y$ is also equal to the pseudorapidity

$$
y=-\ln \tan \frac{1}{2} \theta_{\mathrm{cm}} \text {. }
$$

In terms of $x_{1}$ and $x_{2}$,

$$
\begin{aligned}
& y=\frac{1}{2} \log \frac{x_{1}}{x_{2}}, \\
& p_{\mathrm{T}}^{2}=s x_{1} x_{2} .
\end{aligned}
$$


Note that the condition $\theta_{\mathrm{cm}}=90^{\circ}$ is equivalent to $y=0$ and $x_{1}=x_{2}=p_{\mathrm{T}} / \sqrt{ } \mathrm{s}$.

We use the quark-quark scattering model of Berman, Bjorken, and Kogut [11] to write down the cross section for inclusive scattering. This involves expressing the inclusive cross section as a sum over elementary parton scatterings. Consider the scattering of partons: $i+j \rightarrow i^{\prime}+j^{\prime}$. We associate the invariants $\hat{s}, \hat{t}$, and $\hat{u}$ with this process and define $\hat{x}_{1}=-\hat{u} / \hat{s}$ and $\hat{x}_{2}=-\hat{t} / \hat{s}$. Let $q_{i}^{\mathrm{A}}\left(x_{\mathrm{A}}\right)\left(q_{j}^{\mathrm{B}}\left(x_{\mathrm{B}}\right)\right)$ be the probability of finding parton $i(j)$ with momentum fraction $x_{\mathrm{A}}\left(x_{\mathrm{B}}\right)$ in particle $\mathrm{A}(\mathrm{B})$. After the scattering, parton $i^{\prime}$ fragments into hadron C. Let $D_{i^{\prime}}^{\mathrm{C}}(z)$ be the probability of finding hadron $\mathrm{C}$ with momentum fraction $z$ in parton $\mathrm{i}^{\prime}$. Since $D_{\mathrm{i}^{\prime}}^{\mathrm{C}}(z)$ is expected [14] to behave like $1 / z$ near $z=0$, we define:

$$
G_{\mathbf{i}^{\prime} \mathrm{C}}(z)=z D_{\mathbf{i}^{\prime}}^{\mathrm{C}}(z) \text {. }
$$

We can then write the inclusive cross section as follows:

$$
\mathrm{d} \sigma=\sum_{\mathrm{iji}} \frac{\mathrm{d} \sigma}{\mathrm{d} \hat{x}_{1} \mathrm{~d} \hat{x}_{2}} \mathrm{~d} \hat{x}_{1} \mathrm{~d} \hat{x}_{2} q_{\mathrm{i}}^{\mathrm{A}}\left(x_{\mathrm{A}}\right) q_{\mathrm{j}}^{\mathrm{B}}\left(x_{\mathrm{B}}\right) \frac{G_{\mathrm{i}^{\prime} \mathrm{C}}(z)}{z} \mathrm{~d} x_{\mathrm{A}} \mathrm{d} x_{\mathrm{B}} \mathrm{d} z,
$$

where $\mathrm{d} \sigma / \mathrm{d} \hat{x}_{1} \mathrm{~d} \hat{x}_{2}$ is the cross section for the elementary parton scattering. Note that we must necessarily have $\hat{s}+\hat{t}+\hat{u}=0$ (neglecting parton masses) which is equivalent to $\hat{x}_{1}+\hat{x}_{2}=1$. Hence, we may write:

$$
\frac{\mathrm{d} \sigma}{\mathrm{d} \hat{x}_{1} \mathrm{~d} \hat{x}_{2}}=F\left(\hat{x}_{1}, \hat{x}_{2}\right) \delta\left(\hat{x}_{1}+\hat{x}_{2}-1\right) \text {. }
$$

Furthermore, it is $x_{1}$ and $x_{2}$ which we measure in an experiment. Neglecting parton masses, it follows that:

$$
\begin{aligned}
& \hat{s}=x_{\mathrm{A}} x_{\mathrm{B}} s, \\
& x_{1}=\hat{x}_{1} x_{\mathrm{A}} z, \\
& x_{2}=\hat{x}_{2} x_{\mathrm{B}} z .
\end{aligned}
$$

Using eqs. (A.7)-(A.9), we obtain:

$$
\begin{aligned}
& E \frac{\mathrm{d}^{3} \sigma}{\mathrm{d} p^{3}}=\frac{1}{\pi s} \frac{\mathrm{d} \sigma}{\mathrm{d} x_{1} \mathrm{~d} x_{2}}=\frac{1}{\pi s} \sum_{\mathrm{iji}} \int F\left(\frac{x_{\mathrm{B}} x_{1}}{x_{\mathrm{B}} x_{1}+x_{\mathrm{A}} x_{2}}, \frac{x_{\mathrm{A}} x_{2}}{x_{\mathrm{B}} x_{1}+x_{\mathrm{A}} x_{2}}\right) \\
& \quad \times q_{\mathrm{i}}^{\mathrm{A}}\left(x_{\mathrm{A}}\right) q_{\mathrm{j}}^{\mathrm{B}}\left(x_{\mathrm{B}}\right) G_{\mathrm{i}^{\prime} \mathrm{C}}(z) \frac{x_{\mathrm{A}} x_{\mathrm{B}}}{\left(x_{\mathrm{A}} x_{2}+x_{1} x_{\mathrm{B}}\right)^{2}} \delta\left(z-\frac{x_{1} x_{\mathrm{B}}+x_{2} x_{\mathrm{A}}}{x_{\mathrm{A}} x_{\mathrm{B}}}\right) \\
& \quad \times \mathrm{d} x_{\mathrm{A}} \mathrm{d} x_{\mathrm{B}} \mathrm{d} z .
\end{aligned}
$$

The limits of integration are $0 \leqslant x_{\mathrm{A}}, x_{\mathrm{B}}, z \leqslant 1$.

\section{A.2. The weak-interaction contribution}

In eq. (A.10), the summation over $i, j, i^{\prime}$ instructs us to sum over all possible scatterings. In principle, the elementary parton cross section $F\left(\hat{x}_{1}, \hat{x}_{2}\right)$ contains all 
possible interactions leading to a given parton scattering. In this paper, we are concerned with the weak-interaction contribution to inclusive scattering. In particular, we are interested in the energy region above the threshold for the production of the intermediate vector bosons. Once we are above the $\mathrm{W}$ threshold, i.e., in the region where $s>m_{\mathrm{W}}^{2}$ and $p_{\mathrm{T}}<\frac{1}{2} m_{\mathrm{W}}$; quark-antiquark annihilation into a (real) $\mathrm{W}$ boson dominates all other weak-interaction processes. Therefore, we will assume from now on that we are in this kinematic region, and we will neglect all contributions other than the annihilation process.

Consider the most general V, A structure $g \gamma_{\mu}\left(g_{\mathrm{A}}+g_{\mathrm{A}} \gamma_{5}\right)$ by which a weak vector boson can couple to quarks. We will denote such a vector boson by $\mathrm{V}$ with mass $m_{\mathrm{V}}$. We will usually have the Weinberg-Salam model [32] in mind, which contains two charged $\mathrm{W}$ bosons and one neutral $\mathrm{Z}$ boson. The coupling of the $\mathrm{W}$ to fermions is pure $\mathrm{V}-\mathrm{A}$, corresponding to $g_{\mathrm{A}}=g_{\mathrm{V}}=1$. This allows us to identify $g^{2} / m_{\mathrm{W}}^{2}=G_{\mathrm{F}} / \sqrt{ } 2$. In the simplest version of the Weinberg-Salam model, the coupling of $\mathrm{Z}$ to fermions is given by

$$
\begin{aligned}
& g_{\mathrm{V}}=\frac{2 \sqrt{2}\left(\sin ^{2} \theta_{\mathrm{w}} Q-\frac{1}{2} T_{3}\right)}{\cos \theta_{\mathrm{w}}}, \\
& g_{\mathrm{A}}=\frac{-\sqrt{2} T_{3}}{\cos \theta_{\mathrm{w}}},
\end{aligned}
$$

for a fermion of charge $Q$ and weak isospin $T$. The mass of the $\mathrm{Z}$ is given by $m_{\mathrm{W}}$ $=m_{\mathrm{Z}} \cos \theta_{\mathrm{w}}$. Using the value $\sin ^{2} \theta_{\mathrm{w}}=\frac{1}{4}$ currently favored by neutrino experiments [33], this model predicts $m_{\mathrm{W}}=75 \mathrm{GeV}$ and $m_{\mathrm{Z}}=87 \mathrm{GeV}$; these are the values we use in this paper.

We have worked out the cross section for the diagram in fig. 23. The result is:

$$
F\left(\hat{x}_{1}, \hat{x}_{2}\right)=\frac{G_{\mathrm{F}}^{2} m_{\mathrm{W}}^{4} \hat{s}}{2 \pi\left[\left(\hat{s}-m_{\mathrm{V}}^{2}\right)^{2}+m_{\mathrm{V}}^{2} \Gamma^{2}\right]}\left[C_{1}\left(1-\hat{x}_{2}\right)^{2}+C_{2} \hat{x}_{2}^{2}\right],
$$

where,

$$
\begin{aligned}
& C_{1}=\frac{1}{8}\left[\left(g_{\mathrm{A}}^{2}+g_{\mathrm{V}}^{2}\right)\left(g_{\mathrm{A}}^{\prime 2}+g_{\mathrm{V}}^{\prime 2}\right)+4 g_{\mathrm{A}} g_{\mathrm{A}}^{\prime} g_{\mathrm{V}} g_{\mathrm{V}}^{\prime}\right], \\
& C_{2}=\frac{1}{8}\left[\left(g_{\mathrm{A}}^{2}+g_{\mathrm{V}}^{2}\right)\left(g_{\mathrm{A}}^{\prime 2}+g_{\mathrm{V}}^{\prime 2}\right)-4 g_{\mathrm{A}} g_{\mathrm{A}}^{\prime} g_{\mathrm{V}} g_{\mathrm{V}}^{\prime}\right] .
\end{aligned}
$$

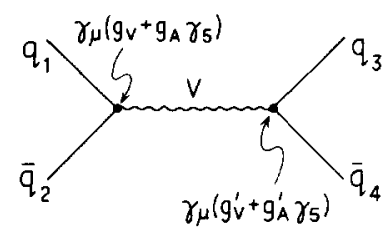

Fig. 23. Quark-antiquark scattering via an s-channel intermediate vector boson. We assume the most general V, A coupling at each vertex. 
Table 5

$C_{1}$ and $C_{2}$ for $Z^{0}$ exchange

\begin{tabular}{lll}
\hline & $C_{1}$ & $C_{2}$ \\
\hline $\mathrm{q}_{2} \overline{\mathrm{q}}_{2} \rightarrow \mathrm{q}_{2} \overline{\mathrm{q}}_{2}$ & 0.0933 & 0.0439 \\
$\mathrm{q}_{1} \overline{\mathrm{q}}_{1} \rightarrow \mathrm{q}_{1} \overline{\mathrm{q}}_{1}$ & 0.2147 & 0.0171 \\
$\mathrm{q}_{1} \overline{\mathrm{q}}_{1} \rightarrow \mathrm{q}_{2} \overline{\mathrm{q}}_{2}$ & 0.1385 & 0.0398 \\
$\mathrm{q}_{2} \overline{\mathrm{q}}_{2} \rightarrow \mathrm{q}_{1} \overline{\mathrm{q}}_{1}$ & 0.1385 & 0.0398
\end{tabular}

Consider $\mathrm{q} \overline{\mathrm{q}} \rightarrow \mathrm{q} \overline{\mathrm{q}}$ via s-channel $\mathrm{Z}$ exchange. $C_{1}$ and $C_{2}$ are defined by eq. (A.13). Let $\mathrm{q}_{2}$ be a charge $+\frac{2}{3}$ quark and let $q_{1}$ be a charge $-\frac{1}{3}$ quark. Using $\sin ^{2} \theta_{w}=\frac{1}{4}$, we obtain the above.

The unprimed (primed) variables refer to the incoming (outgoing) vertex. We have accounted for a non-zero total width $\Gamma$ for the vector boson $V$ by using a BreitWigner denominator. The partial width of $\mathrm{V}$ into two fermions is easily calculated:

$$
\Gamma(\mathrm{V} \rightarrow \overline{\mathrm{ff}})=\frac{G_{\mathrm{F}} m_{\mathrm{W}}^{2} m_{\mathrm{V}}}{12 \pi \sqrt{ } 2}\left(g_{\mathrm{V}}^{2}+g_{\mathrm{A}}^{2}\right) .
$$

One usually assumes that the total width is obtained by summing over all possible lepton and quark pairs. For example, in a model with $2 L$ leptons and $2 Q$ quark flavors, the width of the $\mathrm{W}^{+}$is given by $\Gamma=(3 Q+L) \Gamma(\mathrm{W} \rightarrow \overline{\mathrm{ff}})$, (the 3 is a color factor). In this paper, we have chosen $\Gamma=1 \mathrm{GeV}$ as a typical value for all weak vector bosons. However, $\Gamma$ could be larger, given the current proliferation of new leptons and quarks.

Consider once again the Weinberg-Salam model. The coupling of the charged $W$ to quarks is pure $\mathrm{V}-\mathrm{A}$; hence, we find $C_{1}=1$ and $C_{2}=0$. The coupling of the $\mathrm{Z}$ to quarks is given by eq. (A.11). Using $\sin ^{2} \theta_{w}=\frac{1}{4}$, we obtain numerical results for $C_{1}$ and $C_{2}$ given in table 5 .

We now write out explicitly the formula obtained from eq. (A.10) in the case of W exchange $\left(C_{1}=1, C_{2}=0\right)$. Except near the kinematic boundaries of the process of annihilation into a real $\mathrm{W}$, it is quite accurate to use the narrow width approximation:

$$
\left[\left(\hat{s}-m_{\mathrm{W}}^{2}\right)^{2}+\Gamma^{2} m_{\mathrm{W}}^{2}\right]^{-1} \approx \frac{\pi}{m_{\mathrm{W}} \Gamma} \delta\left(\hat{s}-m_{\mathrm{W}}^{2}\right) .
$$

We then find:

$$
\begin{aligned}
& E \frac{\mathrm{d}^{3} \sigma}{\mathrm{d} p^{3}}=\frac{G_{\mathrm{F}}^{2} m_{\mathrm{W}}^{3} x_{1}^{2}}{2 \pi s \Gamma} \sum_{\mathrm{iji}} \int \frac{x_{\mathrm{A}} x_{\mathrm{B}}^{4}}{\left(x_{1} x_{\mathrm{B}}+x_{2} x_{\mathrm{A}}\right)^{4}} q_{\mathrm{i}}^{\mathrm{A}}\left(x_{\mathrm{A}}\right) q_{\mathrm{j}}^{\mathrm{B}}\left(x_{\mathrm{B}}\right) \\
& \quad \times G_{\mathrm{i}^{\prime} \mathrm{C}}(z) \delta\left(x_{\mathrm{B}}-\frac{m_{\mathrm{W}}^{2}}{x_{\mathrm{A}} s}\right) \delta\left(z-\frac{x_{1} x_{\mathrm{B}}+x_{2} x_{\mathrm{A}}}{x_{\mathrm{A}} x_{\mathrm{B}}}\right) \mathrm{d} x_{\mathrm{A}} \mathrm{d} x_{\mathrm{B}} \mathrm{d} z .
\end{aligned}
$$


We now consider various situations in which we can apply eq. (A.16). We will assume $W$ exchange unless otherwise stated.

(i) Single lepton production: $\mathrm{A}+\mathrm{B} \rightarrow \mu^{ \pm}+\mathrm{X}$ via an intermediate $\mathrm{W}^{ \pm}$. In this case, the outgoing parton does not fragment. Therefore, we put:

$$
G_{\mathrm{i}^{\prime} \mathrm{C}}(z)=\frac{1}{3} z \delta(1-z) \text {. }
$$

The factor of $\frac{1}{3}$ is due to the fact that we must average over initial colors, but there is no color in the final state. We then obtain:

$$
\begin{aligned}
& E \frac{\mathrm{d}^{3} \sigma}{\mathrm{d} p^{3}}=\frac{G_{\mathrm{F}}^{2} m_{\mathrm{W}}^{3} x_{1}^{2} x_{2}}{6 \pi \Gamma} \int_{x_{1} /\left(1-x_{2}\right)}^{1} \mathrm{~d} x_{\mathrm{A}} \delta\left(\frac{s x_{\mathrm{A}}^{2} x_{2}}{x_{\mathrm{A}}-x_{1}}-m_{\mathrm{W}}^{2}\right) \\
& \quad \times \sum_{\mathrm{ij}} \frac{q_{\mathrm{i}}^{\mathrm{A}}\left(x_{\mathrm{A}}\right) q_{\mathrm{j}}^{\mathrm{B}}\left(x_{\mathrm{A}} x_{2} /\left(x_{\mathrm{A}}-x_{1}\right)\right)}{\left(x_{\mathrm{A}}-x_{1}\right)^{2}} .
\end{aligned}
$$

We can integrate over the $\delta$-function. The result is:

$$
E \frac{\mathrm{d}^{3} \sigma}{\mathrm{d} p^{3}}=\frac{G_{\mathrm{F}}^{2} m_{\mathrm{W}}^{3} x_{1}^{2}}{6 \pi \Gamma s} \sum_{\mathrm{ij}} \sum_{k= \pm} \frac{q_{\mathrm{i}}^{\mathrm{A}}\left(y_{k}\right) q_{\mathrm{j}}^{\mathrm{B}}\left[y_{k} x_{2} /\left(y_{k}-x_{1}\right)\right]}{y_{k}\left|y_{k}-2 x_{1}\right|},
$$

where the sum over $k$ is over the roots (denoted by $y_{ \pm}$) of the equation obtained by setting the argument of the $\delta$-function equal to zero, with the requirement that they lie within the limits of integration. Explicitly, we have

$$
y_{ \pm}=\frac{m_{\mathrm{W}}^{2}}{2 x_{2} s}\left[1 \pm \sqrt{1-4 x_{1} x_{2} s / m_{\mathrm{W}}^{2}}\right] \text {. }
$$

The above requirement leads to various cases which are displayed in table 6 . The columns marked $y_{-}$and $y_{+}$answer the question whether $y_{ \pm}$lie within the limits of integration. If the answer is yes, then they contribute to the sum over $k$ in eq. (A.18).

As an example, consider the case of $\theta_{\mathrm{cm}}=90^{\circ}$. Then, we learn that the cross section is zero for $x_{1} /\left(1-x_{2}\right) \leqslant m_{\mathrm{W}}^{2} / s$. In reality, of course, the cross section is not zero; we have obtained zero by virtue of the narrow-width approximation of eq. (A.15). Nevertheless, the cross section in this region will be much smaller than in the region where both the roots $y_{ \pm}$do contribute to the sum (in eq. (A.18)); i.e., for $m_{\mathrm{W}}^{2} \sqrt{s} /\left(s+m_{\mathrm{W}}^{2}\right) \leqslant p_{\mathrm{T}} \leqslant \frac{1}{2} m_{\mathrm{W}}$.

(ii) Total $W$ production cross section $\left(\sigma_{\mathrm{W}}\right)$ : As to check that eq. (A.18) is correct, we can integrate the right-hand side over $x_{1}$ and $x_{2}$. This will give $B \sigma_{W}$ where $B \equiv \Gamma(\mathrm{W} \rightarrow \overline{\mathrm{ff}}) / \Gamma$. The result is:

$$
\sigma_{\mathrm{W}}=\frac{G_{\mathrm{F}}^{2} m_{\mathrm{W}}^{5}}{18 B \Gamma s} \sum_{\mathrm{ij}} \int_{m_{\mathrm{W}}^{2} / s}^{1} \frac{\mathrm{d} x_{\mathrm{A}}}{x_{\mathrm{A}}} q_{\mathrm{i}}^{\mathrm{A}}\left(x_{\mathrm{A}}\right) q_{\mathrm{j}}^{\mathrm{B}}\left(m_{\mathrm{W}}^{2} / x_{\mathrm{A}} s\right) .
$$

Using eq. (A.14), we obtain:

$$
\sigma_{\mathrm{W}}=\frac{\pi \sqrt{2} G_{\mathrm{F}} m_{\mathrm{W}}^{2}}{3 s} \sum_{\mathrm{ij}} \int_{m_{\mathrm{W}}^{2} / s}^{1} \frac{\mathrm{d} x_{\mathrm{A}}}{x_{\mathrm{A}}} q_{\mathrm{i}}^{\mathrm{A}}\left(x_{\mathrm{A}}\right) q_{\mathrm{j}}^{\mathrm{B}}\left(m_{\mathrm{W}}^{2} / x_{\mathrm{A}} s\right),
$$


Table 6

Regions of $x_{1}$ and $x_{2}$

\begin{tabular}{|c|c|c|c|c|}
\hline Case $A: x_{1}\left(1-x_{1}\right) \geqslant x_{2}\left(1-x_{2}\right)$ & $x_{\min }$ & $x_{\max }$ & $y_{-}$ & $y_{+}$ \\
\hline $4 x_{1} x_{2}<\frac{m_{\mathrm{W}}^{2}}{s}<\frac{x_{2}}{1-x_{1}} ; x_{1}<\frac{1}{2}$ & $y_{-}$ & $y_{+}$ & YES & YES \\
\hline$\frac{x_{2}}{1-x_{1}} \leqslant \frac{m_{\mathrm{W}}^{2}}{s} \leqslant \frac{x_{1}}{1-x_{2}}$ & $y_{-}$ & 1 & YES & NO \\
\hline$\frac{x_{1}}{1-x_{2}} \leqslant \frac{m_{W}^{2}}{s} \leqslant 1$ & $\frac{m_{\mathrm{W}}^{2}}{s}$ & 1 & NO & NO \\
\hline Case $B: x_{1}\left(1-x_{1}\right)<x_{2}\left(1-x_{2}\right)$ & $x_{\min }$ & $x_{\max }$ & $y_{-}$ & $y_{+}$ \\
\hline $4 x_{1} x_{2}<\frac{m_{\mathrm{W}}^{2}}{s} \leqslant \frac{x_{1}}{1-x_{2}} ; x_{2} \leqslant \frac{1}{2}$ & $y_{-}$ & $y_{+}$ & YES & YES \\
\hline$\frac{x_{1}}{1-x_{2}} \leqslant \frac{m_{\mathrm{W}}^{2}}{s} \leqslant \frac{x_{2}}{1-x_{1}}$ & $\frac{m_{\mathrm{W}}^{2}}{s}$ & $y_{+}$ & NO & YES \\
\hline$\frac{x_{2}}{1-x_{1}} \leqslant \frac{m_{W}^{2}}{s} \leqslant 1$ & $\frac{m_{\mathrm{W}}^{2}}{s}$ & 1 & NO & NO \\
\hline
\end{tabular}

Assume that $4 p_{\mathrm{T}}^{2} \leqslant m_{\mathrm{W}}^{2} \leqslant s$. Then, since $x_{1}+x_{2} \leqslant 1$, we have two basic cases to consider. We define $y_{ \pm}$in eq. (A.19). Then, under the column marked $y_{ \pm}$, we answer the question: is $x_{1} /\left(1-x_{2}\right)<y_{ \pm}<1$ true? The columns marked $x_{\min } ; x_{\max }$ give the limits of integration in eq. (A.22).

which is the Drell-Yan formula [26,1] for W production.

(iii) Inclusive hadron production: we may integrate eq. (A.16) over the two $\delta$-functions to obtain:

$$
E \frac{\mathrm{d}^{3} \sigma}{\mathrm{d} p^{3}}=\frac{G_{\mathrm{F}}^{2} m_{\mathrm{W}}^{3}}{2 \pi \Gamma s x_{1}^{2}} \sum_{\mathrm{iji}} \int_{x_{\min }}^{x_{\max }} \frac{x q_{\mathrm{i}}^{\mathrm{A}}(x) q_{\mathrm{j}}^{\mathrm{B}}\left(m_{\mathrm{W}}^{2} / x s\right) G_{\mathrm{i}^{\prime} \mathrm{C}}(z) \mathrm{d} x}{\left(1+x^{2} x_{2} s / x_{1} m_{\mathrm{W}}^{2}\right)^{4}},
$$

where $z \equiv\left(m_{\mathrm{W}}^{2} x_{1}+x^{2} x_{2} s\right) / m_{\mathrm{W}}^{2} x$ and $x_{\min }, x_{\max }$ are given in table 6 for the various cases.

Eq. (A.22) as it stands is slightly misleading. Thus, we will demonstrate its use by considering an example: $\overline{\mathrm{p}} \mathrm{p} \rightarrow \rho^{+}+\mathrm{X}$. In the limit of zero Cabibbo angle, we need to consider the four diagrams in fig. 2. By convention, we choose the proton to be particle A and the $\rho$ meson to be particle C (see subsect. A.1). Thus, eq. (A.22) is only valid for diagrams (a) and (b) in fig. 2 . The reason is that we have derived $F\left(\hat{x}_{1}, \hat{x}_{2}\right)$ for the case of $\mathrm{q} \overline{\mathrm{q}} \rightarrow \mathrm{q} \overline{\mathrm{q}}$ where $\hat{t}=-\left(p_{i^{\prime}}-p_{\mathrm{i}}\right)^{2}$ and both $i$ and $i^{\prime}$ are quarks (or both 
antiquarks). Diagrams (c) and (d) in fig. 2 correspond to the case where $i$ and $i^{\prime}$ are a quark and antiquark. If we denote this process by $\mathrm{q} \overline{\mathrm{q}} \rightarrow \overline{\mathrm{q}} \mathrm{q}$ then it is clear that

$$
\left.F\left(\hat{x}_{1}, \hat{x}_{2}\right)\right|_{\mathrm{q} \overline{\mathrm{q}} \rightarrow \overrightarrow{\mathrm{q}} \mathrm{q}}=\left.F\left(\hat{x}_{2}, \hat{x}_{1}\right)\right|_{\mathrm{q} \overline{\mathrm{q}} \rightarrow \mathrm{q} \overline{\mathrm{q}}} .
$$

One can see by examining eq. (A.10) that, in the case of $q \bar{q} \rightarrow \bar{q} q$, we must modify eq. (A.22) by making the interchange $\mathrm{A} \leftrightarrow \mathrm{B}$ and $x_{1} \leftrightarrow x_{2}$. In the example of $\overline{\mathrm{p}} \mathrm{p} \rightarrow \rho^{+}+\mathrm{X}$, we find

$$
\begin{aligned}
& E \frac{\mathrm{d}^{3} \sigma}{\mathrm{d} p^{3}}\left(\overline{\mathrm{p} p} \rightarrow \rho^{+}+\mathrm{X}\right) \\
& =\frac{G_{\mathrm{F}}^{2} m_{\mathrm{W}}^{3}}{2 \pi \Gamma s x_{1}^{2}} \int_{x_{\min }}^{x_{\max }} \frac{x \mathrm{~d} x}{\left(1+x^{2} x_{2} s / x_{1} m_{\mathrm{W}}^{2}\right)^{4}} \\
& \quad \times\left[u(x) d\left(m_{\mathrm{W}}^{2} / x s\right) G_{\mathrm{u} \rho^{+}}(z)+\bar{d}(x) \bar{u}\left(m_{\mathrm{W}}^{2} / x s\right) G_{\overline{\mathrm{d}} \rho^{+}}(z)\right] \\
& \quad+\frac{G_{\mathrm{F}}^{2} m_{\mathrm{W}}^{3}}{2 \pi \Gamma s x_{2}^{2}} \int_{x_{\min }^{\prime}}^{x_{\max }^{\prime}} \frac{x \mathrm{~d} x}{\left(1+x^{2} x_{1} s / x_{2} m_{\mathrm{W}}^{2}\right)^{4}} \\
& \quad \times\left[\bar{u}(x) \bar{d}\left(m_{\mathrm{W}}^{2} / x s\right) G_{\mathrm{u} \rho^{+}}\left(z^{\prime}\right)+d(x) u\left(m_{\mathrm{W}}^{2} / x s\right) G_{\overline{\mathrm{d}} \rho^{+}}\left(z^{\prime}\right)\right],
\end{aligned}
$$

where $x_{\min }^{\prime}, x_{\max }^{\prime}$, and $z^{\prime}$ are obtained from $x_{\min }, x_{\max }$ and $z$ by interchanging $x_{1}$ and $x_{2}$ (cf. eq. (A.22)). The four terms above correspond to the four diagrams in fig. 2 , respectively. Note that in the first term, $u(x)$ is the probability of finding a u-quark inside a proton, and $d\left(m_{\mathrm{W}}^{2} / x s\right)$ is the probability of finding a $\overline{\mathrm{d}}$-quark inside an antiproton etc.

\section{A.3. Quark functions}

There have been a number of parametrizations of the quark distribution functions in the literature [34]. Our results are very insensitive to the effects of changing from one set of quark functions to another; we have used those of Barger and Phillips.

Denoting the valence-quark distributions by $u_{\mathrm{V}}(x)$ and $d_{\mathrm{V}}(x)$ and denoting the seaquark distributions by $s(x)$,

$$
\begin{aligned}
& u(x)=u_{\mathrm{V}}(x)+s(x), \\
& d(x)=d_{\mathrm{V}}(x)+s(x), \\
& \bar{u}(x)=\bar{d}(x)=s(x),
\end{aligned}
$$

where

$$
\begin{aligned}
& u_{V}(x)=0.594 x^{-1 / 2}\left(1-x^{2}\right)^{3}+0.461 x^{-1 / 2}\left(1-x^{2}\right)^{5} \\
& +0.621 x^{-1 / 2}\left(1-x^{2}\right)^{7}
\end{aligned}
$$




$$
\begin{aligned}
& d_{\mathrm{V}}(x)=0.072 x^{-1 / 2}\left(1-x^{2}\right)^{3}+0.206 x^{-1 / 2}\left(1-x^{2}\right)^{5} \\
& \quad+0.621 x^{-1 / 2}\left(1-x^{2}\right)^{7} \\
& s(x)=0.145 x^{-1}(1-x)^{9} .
\end{aligned}
$$

Since in this paper we always work in the approximation of zero Cabibbo angle, we need not worry about strange quarks in the sea (assuming we can safely neglect the charm quarks in the sea).

We also want to calculate cross sections involving polarized beams. This will require "polarized" quark functions; i.e., the probability of finding a quark of a given helicity in a polarized proton. There are a number of models in the literature [35-38] for the polarized quark functions. We have used results given by Kaur [37]. The notation is as follows: Let $u_{+}(x)$ be the probability of finding a positive helicity u-quark with momentum fraction $x$ inside a positive helicity proton. Note that $u_{+}(x)$ is also the probability of finding a negative helicity u-quark inside a negative helicity proton (and the probability of finding a positive helicity $\overline{\mathrm{u}}$-quark inside a positive helicity antiproton, etc.). Similarly, $u_{-}(x)$ is the probability of finding a negative helicity u-quark inside a positive helicity proton. We define $d_{ \pm}(x)$ in an analogous manner. Clearly,

$$
\begin{aligned}
& u_{+}(x)+u_{-}(x)=u(x) \\
& d_{+}(x)+d_{-}(x)=d(x)
\end{aligned}
$$

The sea quarks are assumed to be unpolarized:

$$
s_{+}(x)=s_{-}(x)=\frac{1}{2} s(x) \text {. }
$$

Using this notation, Kaur's results can be stated as follows:

$$
\begin{aligned}
& u_{+}(x)=\frac{1}{2} u(x)[1+f(x)]-\frac{1}{3} d(x) f(x)-\frac{1}{6} s(x) f(x), \\
& u_{-}(x)=u(x)-u_{+}(x) \\
& d_{+}(x)=\frac{1}{2} d(x)\left[1-\frac{1}{3} f(x)\right]+\frac{1}{6} s(x) f(x) \\
& d_{-}(x)=d(x)-d_{+}(x)
\end{aligned}
$$

where $f(x)$ is what Kaur calls the spin dilution factor:

$$
f(x)=\left[1+H_{0} x^{-1 / 2}(1-x)^{2}\right]^{-1},
$$

and $H_{0}=0.052$. ( $H_{0}$ is adjusted so that the Bjorken sum rule is satisfied by the quark functions.) Note that as $x \rightarrow 0, u_{+}(x)=u_{\bar{v}}(x)=\frac{1}{2} u(x)$ and $d_{+}(x)=d_{-}(x)=\frac{1}{2} d(x)$. As $x \rightarrow 1$ we approach the SU(6) limit: $u_{+} \overline{\mathrm{V}}=\frac{5}{6} u^{\mathrm{V}}$ and $d_{+}^{\mathrm{V}}=\frac{1}{3} d^{\mathrm{V}}$, if we use the fact that $d^{\mathrm{V}}=\frac{1}{2} u^{\mathrm{V}}$ (i.e., there are twice as many valence $\mathrm{u}$-quarks in a proton as compared with valence d-quarks). 


\section{A.4. The quark fragmentation function}

Using eqs. (A.22) and (A.23), we can calculate the cross section for meson and baryon production, if we know the functions $G_{\mathrm{i}^{\prime} \mathrm{C}}(z)$ (corresponding to hadron C). Detailed studies $[13,14,39]$ have been made of these functions by applying the parton model to electroproduction and large- $p_{\mathrm{T}}$ hadronic scattering. In this paper, we need only some gross properties of these functions. We therefore make use of the following simple model. We write [13]

$$
G_{\mathrm{i}^{\prime} \mathrm{C}}(z)=K_{\mathrm{i}^{\prime}}^{\mathrm{C}} G(z) \text {. }
$$

We will use some universal function $G(z)$ for all the light mesons. (For baryons and charmed mesons, we will allow for the possibility of choosing a different function.) Since [11]

$$
\sum_{C} \int_{0}^{1} G_{\mathrm{i}^{\prime} \mathrm{C}}(z) \mathrm{d} z=1,
$$

it follows that (if we normalize $G(z)$ to 1 ):

$$
\sum_{\mathrm{C}} K_{\mathrm{i}^{\prime}}^{\mathrm{C}}=1
$$

We make the following approximations:

(i) We use SU(6) wave functions to obtain the constants $K_{\mathbf{i}^{\prime}}^{\mathrm{C}}$.

(ii) We only consider the decay of parton $i$ ' into the "ground state" hadrons, i.e., pseudoscalar and vector mesons and the $\frac{1}{2}^{+}$and $\frac{3}{2}^{+}$baryons. All higher SU(6) multiplets are neglected.

(iii) If $\mathrm{B}$ is a baryon and $\mathrm{M}$ is a meson, $K_{\mathrm{i}^{\prime}}^{\mathrm{B}}<<K_{\mathrm{i}^{\prime}}^{\mathrm{M}}$ so that:

$$
\sum_{\mathrm{M}} K_{\mathrm{i}^{\prime}}^{\mathrm{M}} \approx 1
$$

(iv) A parton $i^{\prime}$ decays into a meson by combining with another parton created out of the vacuum. We assume that the creation of $u \bar{u}$ and $d \bar{d}$ pairs are equally likely; we associate a suppression factor $R_{1}$ for the creation of $s \bar{s}$ pairs as compared with $u \bar{u}$ and $d \bar{d}$.

(v) In order for a parton $i^{\prime}$ to decay into a baryon, two $q \bar{q}$ pairs must be created out of the vecuum. We associate a suppression factor $R_{2}$ for the decay of a parton into a baryon as compared to a meson.

In this paper, we use $R_{1}=0.5[15]$ and $R_{2}=0.1$ [40] as suggested by some authors.

(vi) We assume that large $-p_{\mathrm{T}}$ hadrons with reasonably large momentum originated from parton $i^{\prime}$ and remember its helicity. Furthermore, the parton $i^{\prime}$ is assumed to be a valence quark inside that hadron. This is our most drastic assumption. Field and Feynman [39] claim that this is true at most 50\% of the time. However, we feel that the results we obtain will not change significantly with a more careful treatment of this problem. 
Table 7

Average number of polarized quarks in a polarized hadron

\begin{tabular}{cccccccccccccc}
\hline & $\lambda^{\prime}$ & $\mathrm{u}_{-}$ & $\mathrm{u}_{+}$ & $\mathrm{d}_{-}$ & $\mathrm{d}_{+}$ & $\mathrm{s}_{-}$ & $\mathrm{s}_{+}$ & $\overline{\mathrm{u}}_{-}$ & $\overline{\mathrm{u}}_{+}$ & $\overline{\mathrm{d}}_{-}$ & $\overline{\mathrm{d}}_{+}$ & $\overline{\mathrm{s}}_{-}$ & $\overline{\mathrm{s}}_{+}$ \\
\hline$\pi^{+}$ & 0 & $\frac{1}{2}$ & $\frac{1}{2}$ & 0 & 0 & 0 & 0 & 0 & 0 & $\frac{1}{2}$ & $\frac{1}{2}$ & 0 & 0 \\
$\rho^{+}$ & +1 & 0 & 1 & 0 & 0 & 0 & 0 & 0 & 0 & 0 & 1 & 0 & 0 \\
& 0 & $\frac{1}{2}$ & $\frac{1}{2}$ & 0 & 0 & 0 & 0 & 0 & 0 & $\frac{1}{2}$ & $\frac{1}{2}$ & 0 & 0 \\
& -1 & 1 & 0 & 0 & 0 & 0 & 0 & 0 & 0 & 1 & 0 & 0 & 0 \\
$\mathrm{~K}^{*+}$ & +1 & 0 & 1 & 0 & 0 & 0 & 0 & 0 & 0 & 0 & 0 & 0 & 1 \\
& 0 & $\frac{1}{2}$ & $\frac{1}{2}$ & 0 & 0 & 0 & 0 & 0 & 0 & 0 & 0 & $\frac{1}{2}$ & $\frac{1}{2}$ \\
& -1 & 1 & 0 & 0 & 0 & 0 & 0 & 0 & 0 & 0 & 0 & 0 & 0 \\
$\varphi^{0}$ & +1 & 0 & 0 & 0 & 0 & 0 & 1 & 0 & 0 & 0 & 0 & 0 & 1 \\
& 0 & 0 & 0 & 0 & 0 & $\frac{1}{2}$ & $\frac{1}{2}$ & 0 & 0 & 0 & 0 & $\frac{1}{2}$ & $\frac{1}{2}$ \\
& -1 & 0 & 0 & 0 & 0 & 1 & 0 & 0 & 0 & 0 & 0 & 1 & 0 \\
\multirow{2}{*}{$\mathrm{p}$} & $\frac{1}{2}$ & $\frac{5}{3}$ & $\frac{1}{3}$ & $\frac{1}{3}$ & $\frac{2}{3}$ & 0 & 0 & 0 & 0 & 0 & 0 & 0 & 0 \\
& $-\frac{1}{2}$ & $\frac{1}{3}$ & $\frac{5}{3}$ & $\frac{2}{3}$ & $\frac{1}{3}$ & 0 & 0 & 0 & 0 & 0 & 0 & 0 & 0 \\
$\Lambda^{0}$ & $+\frac{1}{2}$ & $\frac{1}{2}$ & $\frac{1}{2}$ & $\frac{1}{2}$ & $\frac{1}{2}$ & 0 & 1 & 0 & 0 & 0 & 0 & 0 & 0 \\
& $-\frac{1}{2}$ & $\frac{1}{2}$ & $\frac{1}{2}$ & $\frac{1}{2}$ & $\frac{1}{2}$ & 1 & 0 & 0 & 0 & 0 & 0 & 0 & 0 \\
\hline
\end{tabular}

Using the SU(6) wave functions, we compute the average number of quarks $q_{\lambda}$ with helicity $\lambda$ in a hadron $C_{\lambda^{\prime}}$ with helicity $\lambda^{\prime}$.

To implement these approximations, we first calculate the average number of quarks of helicity $\lambda$ in a hadron of helicity $\lambda^{\prime}$ using the SU(6) wave functions. Let $t_{\mathrm{i}^{\prime}}^{\mathrm{C}}$ be the average number of quarks of type $\mathrm{i}^{\prime}$ in hadron $\mathrm{C}$. The $t_{\mathrm{i}^{\prime}}^{\mathrm{C}}$ for some of the ground-state hadrons are given in table 7. Given approximation (vi) above, the $K_{i^{\prime}}^{\mathrm{C}}$ are proportional to the $t_{\mathrm{i}^{\prime}}^{\mathrm{C}}$ multiplied by the factor $R_{1}$ and/or $R_{2}$ when appropriate. To obtain the actual values of $K_{\mathrm{i}^{\prime}}^{\mathrm{C}}$, we normalize them according to eq. (A.34). In that case, we only have to consider the ground state mesons. If we consider the case where $\mathrm{i}^{\prime}$ is, for example, a $\mathrm{u}_{+}$quark, then $K_{\mathrm{u}_{+}}^{\mathrm{C}}=a r t_{\mathrm{u}_{+}}^{\mathrm{C}}$ where $r=R_{1}$ when $\mathrm{C}$ is a meson with non-zero strangeness and $r=1$ otherwise. To determine the constant $a$, we sum over all the ground-state mesons of all helicities; using eq. (A.34), we find $a=1 /\left(4+2 R_{1}\right)$. For $R_{1}=0.5$, we see that $a=0.2$. Therefore, we have the following rule: in order to obtain $K_{\mathrm{i}^{\prime}}^{\mathrm{C}}$

(1) Take the number given in column $i^{\prime}$ and row $\mathrm{C}$ of table 7 ;

(2) Multiply by $a=0.2$;

(3) If an $s$ or $\bar{s}$ quark is created from the vacuum, multiply by $R_{1}=0.5$;

(4) If $\mathrm{C}$ is a baryon, multiply by $R_{2}=0.1$.

Using these rules, we can reproduce table 1 . For example, a $\rho^{+}$originates from a 


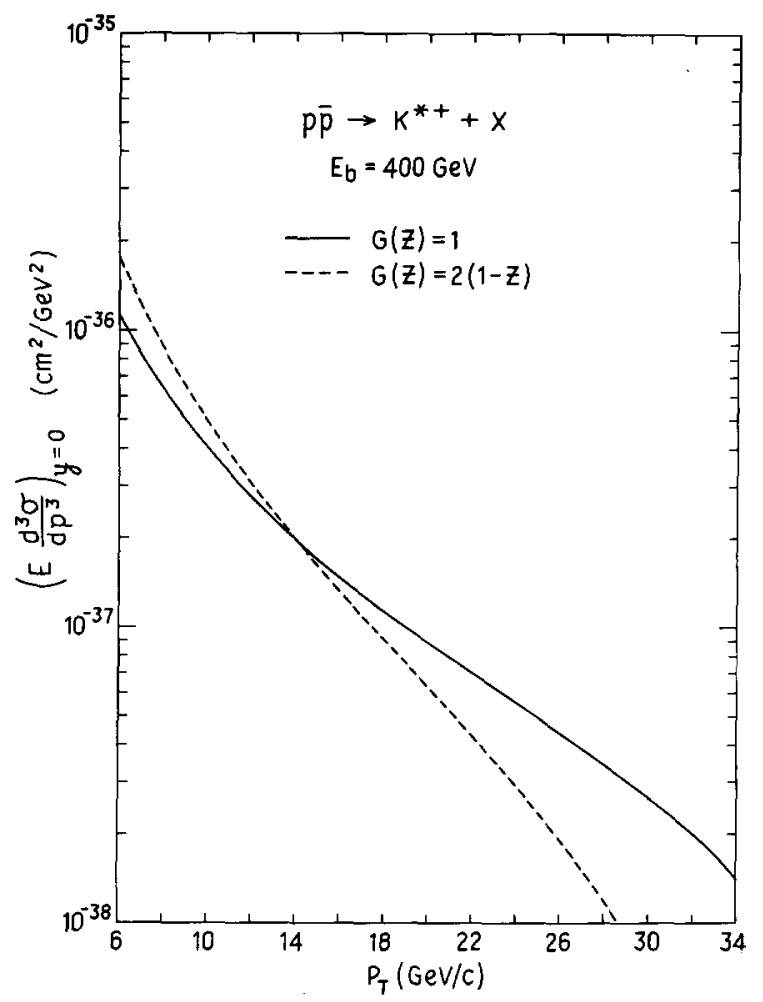

Fig. 24. Comparison of fragmentation functions. We compare the results of two choices of $G(z)$ on the weak inclusive scattering cross section. See eq. (A.31).

u-quark or a d-quark. Using the rules above ${ }^{\star}, K_{\mathrm{u}}^{\rho^{+}}=K_{\overline{\mathrm{d}}}^{\rho^{+}}=0.3$. Similarly, for $\Lambda^{0}$ production, we need to know: $\mathrm{K}_{\mathrm{u}}^{\Lambda^{0}}=\mathrm{K}_{\mathrm{d}}^{\Lambda^{0}}=0.01$ and $\mathrm{K}_{\mathrm{s}}^{\Lambda^{0}}=0.02$. Note that in the case of $\Lambda^{0}$, we are actually calculating the cross section for producing prompt $\Lambda^{0}$ s (i.e., excluding $\Lambda$ 's which result from the decay of other particles).

Using our rules above, we could also calculate inclusive $\pi$ production. Since $\mathrm{K}_{\mathrm{u}}^{\pi^{+}}=0.1$, we obtain the standard SU(6) result that the $\rho / \pi$ ratio should be $3: 1$. (Experimental evidence for this ratio in recent $\mathrm{e}^{+} \mathrm{e}^{-}$data has been noted [41].) However, one must separate out prompt pions in order for this ratio to be valid. Since we are mostly interested in resonance production, we did not study this point in any detail.

Let us now consider the function $G(z)$. It is usually assumed that as $z \rightarrow 1$, $G(z) \sim(1-z)^{p}$ for some positive power $p$. In the region of most interest to us, $10 \leqslant p_{\mathrm{T}} \leqslant 37 \mathrm{GeV} / c$ and $-2 \leqslant y \leqslant 2$, our results are fairly insensitive to the choice

${ }^{\star}$ Note that $K_{i^{\prime}}^{\mathrm{C}}=\Sigma_{\lambda^{\prime}} K_{i^{\prime}(\lambda)}^{C\left(\lambda^{\prime}\right)}$ which is independent of $\lambda$. 
of $G(z)$. We present fig. 24 as evidence of this by comparing the choice of $G(z) \equiv 1$ with $G(z)=2(1-z)$. Significant differences occur only at very large $p_{\mathrm{T}}$ where the cross section is smallest. Therefore, we decided to use $G(z) \equiv 1$ for all our calculations.

\section{A.5. Inclusive production of polarized hadrons}

If parity is conserved, then the number of helicity $+\lambda$ hadrons produced will equal the number of $-\lambda$ hadrons produced (in a collision of unpolarized beams). Thus, a difference in these two numbers signals parity violation and suggests a weak interaction effect. Therefore, we have calculated the weak-interaction contribution to the inclusive production of hadrons of definite helicity. We need to know $F_{\lambda \lambda^{\prime}}\left(\hat{x}_{1}, \hat{x}_{2}\right)$ which is the cross section for $\mathrm{q} \overline{\mathrm{q}} \rightarrow \mathrm{q} \overline{\mathrm{q}} ; \lambda\left(\lambda^{\prime}\right)$ is the helicity of the outgoing quark (antiquark). Neglecting the quark masses, we find:

$$
\begin{aligned}
& F_{++}=F_{--}=0, \\
& F_{+-}\left(\hat{x}_{1}, \hat{x}_{2}\right)=\frac{G_{\mathrm{F}}^{2} m_{\mathrm{W}}^{4} \hat{s}\left(g_{\mathrm{V}}^{\prime}-g_{\mathrm{A}}^{\prime}\right)^{2}}{32 \pi\left(m_{\mathrm{V}}^{2}-\hat{s}\right)^{2}} \\
& \quad \times\left[\left(1-\hat{x}_{2}\right)^{2}\left(g_{\mathrm{V}}-g_{\mathrm{A}}\right)^{2}+\hat{x}_{2}^{2}\left(g_{\mathrm{V}}+g_{\mathrm{A}}\right)^{2}\right], \\
& F_{-+}\left(\hat{x}_{1}, \hat{x}_{2}\right)=\frac{G_{\mathrm{F}}^{2} m_{\mathrm{W}}^{4} \hat{s}\left(g_{\mathrm{V}}^{\prime}+g_{\mathrm{A}}^{\prime}\right)^{2}}{32 \pi\left(m_{\mathrm{V}}^{2}-\hat{s}\right)^{2}} \\
& \quad \times\left[\left(1-\hat{x}_{2}\right)^{2}\left(g_{\mathrm{V}}+g_{\mathrm{A}}\right)^{2}+\hat{x}_{2}^{2}\left(g_{\mathrm{V}}-g_{\mathrm{A}}\right)^{2}\right] .
\end{aligned}
$$

One can now derive the required results by following the steps of subsect. A.2. The quark fragmentation function $G_{\mathrm{q}_{\lambda}} \mathrm{C}_{\lambda^{\prime}}(z)$ can be obtained using the methods of subsect. A.4. Note that for W exchange $\left(g_{\mathrm{V}}=g_{\mathrm{A}}=g_{\mathrm{V}}^{\prime}=g_{\mathrm{A}}^{\prime}=1\right)$, we find that the only contribution is $F_{-+}$; i.e., left-handed quarks and right-handed antiquarks.

It is worth working out an example in detail. Consider $\mathrm{pp} \rightarrow \rho^{ \pm}( \pm 1)+\mathrm{X} v i a \mathrm{~W}^{+}$ exchange. For simplicity, we will assume that the $\rho^{+}$is produced at $\theta_{\mathrm{cm}}=90^{\circ}$, i.e., at $x_{1}=x_{2}=p_{\mathrm{T}} / \sqrt{ } s$. Because the $\mathrm{W}^{+}$decays into $\mathrm{u}_{-} \overline{\mathrm{d}}_{+}$, diagrams $(\mathrm{b})$ and $(\mathrm{d})$ of fig. 2 contribute to the production of $\rho^{+}(+1)$, and diagrams (a) and (c) contribute to the production of $\rho^{+}(-1)$. Using the results of subsect. A.4, $G_{\mathrm{u}(-) \rho^{+}(-1)}(z)=$ $\frac{2}{3} G_{\mathrm{u} \rho^{+}}(z)$ and $G_{\mathrm{d}(+) \rho^{+}(+1)}(z)=\frac{2}{3} G_{\mathrm{d} \rho^{+}}(z)$. We can therefore write down the cross sections for the production of $\rho^{+}( \pm 1)$ with the help of eq. (A.24) (noting that we are dealing here with pp scattering at $\theta_{\mathrm{cm}}=90^{\circ}$ ):

$$
\begin{aligned}
& E \frac{\mathrm{d}^{3} \sigma}{\mathrm{d} p^{3}}\left(\mathrm{pp} \rightarrow \rho^{+}(-1)+\mathrm{X}\right) \\
& \quad=\frac{2 G_{\mathrm{F}}^{2} m_{\mathrm{W}}^{3}}{3 \pi \Gamma p_{\mathrm{T}}^{2}} \int_{x_{\min }}^{x_{\max }} \frac{x \mathrm{~d} x}{\left(1+x^{2} s / m_{\mathrm{W}}^{2}\right)^{4}} u(x) \bar{d}\left(m_{\mathrm{W}}^{2} / x s\right) G_{\mathrm{u} \rho^{+}}(z),
\end{aligned}
$$




$$
\begin{aligned}
& E \frac{\mathrm{d}^{3} \sigma}{\mathrm{d} p^{3}}\left(\mathrm{pp} \rightarrow \rho^{+}(+1)+\mathrm{X}\right) \\
& \quad=\frac{2 G_{\mathrm{F}}^{2} m_{\mathrm{W}}^{3}}{3 \pi \Gamma p_{\mathrm{T}}^{2}} \int_{x_{\min }}^{x_{\max }} \frac{x \mathrm{~d} x}{\left(1+x^{2} s / m_{\mathrm{W}}^{2}\right)^{4}} \bar{d}(x) u\left(m_{\mathrm{W}}^{2} / x s\right) G_{\overline{\mathrm{d}} \rho}+(z) .
\end{aligned}
$$

We can see immediately that the two cross sections are unequal indicating a parity violation. Note that the contributions from diagrams (a) and (c) of fig. 2 turned out to be equal at $\theta_{\mathbf{c m}}=90^{\circ}$; similarly for diagrams (b) and (d). If we had dealt with a parity-conserving theory, then diagrams (a) and (b) would also have been equal (leading to an equality of the two cross sections in eq. (A.36)). In actuality, diagrams (a) and (b) are not equal; this can be attributed to the fact that $F_{+-}\left(\hat{x}_{1}, \hat{x}_{2}\right) \neq F_{-+}\left(\hat{x}_{1}, \hat{x}_{2}\right)$.

\section{A.6. Weak-interaction effects using polarized beams}

If polarized beams are available, parity violation becomes easier to detect. First, let us consider the generalization of the Drell-Yan formula given by eq. (A.21). We must be careful here in our notation. Let us consider the general case of $A+B \rightarrow V$ where $\mathrm{V}$ is a vector boson which couples to quarks via $g \gamma_{\mu}\left(g_{\mathrm{V}}+g_{\mathrm{A}} \gamma_{5}\right) ; g^{2} / m_{\mathrm{W}}^{2}=$ $G_{\mathrm{F}} / \sqrt{ } 2$. Let $u_{+}^{\mathrm{A}_{+}}(x)$ be the probability of finding a helicity $+\frac{1}{2}$ u-quark in a helicity $+\frac{1}{2} \mathrm{~A}$, etc. (Note that $u_{-}^{\mathrm{A}}-(x)=u_{+}^{\mathrm{A}_{+}}(x)$ ). We denote the total cross section for $\mathrm{A}(\lambda)+\mathrm{B}\left(\lambda^{\prime}\right) \rightarrow \mathrm{V}$ by $\sigma_{\lambda \lambda^{\prime}}$. Then, we find:

$$
\begin{aligned}
& \sigma_{\lambda \lambda^{\prime}}=\frac{\pi \sqrt{2} G_{\mathrm{F}} m_{\mathrm{W}}^{2}}{3 s} \int_{m_{\mathrm{V}}^{2} / s}^{1} \frac{\mathrm{d} x}{x} \\
& \quad \times\left\{\left(g_{\mathrm{A}}-g_{\mathrm{V}}\right)^{2}\left[u_{+}^{\mathrm{A} \lambda}(x) \bar{d}_{-}^{\mathrm{B} \lambda^{\prime}}\left(m_{\mathrm{V}}^{2} / x s\right)+u_{+}^{\mathrm{B} \lambda^{\prime}}(x) \bar{d}_{-}^{\mathrm{A} \lambda}\left(m_{\mathrm{V}}^{2} / x s\right)\right]\right. \\
& \left.+\left(g_{\mathrm{A}}+g_{\mathrm{V}}\right)^{2}\left[u_{-}^{\mathrm{A} \lambda}(x) \bar{d}_{+}^{\mathrm{B} \lambda^{\prime}}\left(m_{\mathrm{V}}^{2} / x s\right)+u_{-}^{\mathrm{B} \lambda^{\prime}}(x) \bar{d}_{+}^{\mathrm{A} \lambda}\left(m_{\mathrm{V}}^{2} / x s\right)\right]\right\}
\end{aligned}
$$

(where we have assumed zero Cabibbo angle and have neglected, the heavy quarks in the sea). If we take both $A$ and $B$ to be protons, then we find $\sigma_{+-}=\sigma_{-+}$(which must be true due to identical particles) and $\sigma_{++} \neq \sigma_{-}$. Parity conservation requires $\sigma_{++}=\sigma_{--}$; hence any non-zero difference $\sigma_{++}-\sigma_{--}$is a measure of parity violation, which would indicate the presence of weak-interaction effects. It is important to note that $\sigma_{++} \neq \sigma_{+-}$does not indicate a parity violation. In general, one expects (and sees experimentally [27]) that strong interaction effects lead to $\sigma_{++} \neq \sigma_{+-}$. If we take $A$ to be a proton and B to be an antiproton, then all four $\sigma_{\lambda \lambda^{\prime}}$ are unequal. Note that we have used the fact that $\bar{d}_{-}^{\bar{p}_{+}}(x)=d_{-}(x)$, etc. Again, we emphasize that $\sigma_{++} \neq \sigma_{--}$and $\sigma_{+-} \neq \sigma_{-+}$(in the case of pp scattering) are the only inequalities which signal a parity violation.

One can also detect parity violation by polarizing one beam. In that case, we can 
define

$$
\sigma_{\lambda}=\frac{1}{2} \sum_{\lambda^{\prime}} \sigma_{\lambda \lambda^{\prime}}
$$

Eq. (A.37) then leads to $\sigma_{+} \neq \sigma_{-}$which indicates a parity violation. Note that the total cross section for producing the weak vector boson $\mathrm{V}$ is

$$
\sigma=\frac{1}{4} \sum_{\lambda \lambda^{\prime}} \sigma_{\lambda \lambda^{\prime}},
$$

which reduces to eq. (A.21) when $g_{\mathrm{V}}=g_{\mathrm{A}}=1$.

Suppose one attempted to search for parity violation by the methods given above. One obvious problem is that the strong interaction "background" is overwhelming if we integrate over all of phase space. To improve the weak-interaction signal, we can try triggering only on large- $p_{\mathrm{T}}$ inclusive hadron events. Our event rate will decrease by a large factor; however, the strong "background" will be reduced tremendously. Therefore, we have calculated the weak-interaction cross section for inclusive hadron production by polarized beams. Again, we use the formalism described in previous subsections of this appendix. The new result we need is $F^{\lambda \lambda^{\prime}}\left(\hat{x}_{1}, \hat{x}_{2}\right)$ which is the cross section for $q \bar{q} \rightarrow q \bar{q} ; \lambda\left(\lambda^{\prime}\right)$ is the helicity of the initial quark (antiquark). The result is almost identical to eq. (A.35):

$$
\begin{aligned}
& F^{++}=F^{--}=0, \\
& F^{+-}\left(\hat{x}_{1}, \hat{x}_{2}\right)=\frac{G_{\mathrm{F}}^{2} m_{\mathrm{W}}^{4} \hat{s}\left(g_{\mathrm{V}}-g_{\mathrm{A}}\right)^{2}}{8 \pi\left(m_{\mathrm{V}}^{2}-\hat{s}\right)^{2}} \\
& \quad \times\left[\left(1-\hat{x}_{2}\right)^{2}\left(g_{\mathrm{V}}^{\prime}-g_{\mathrm{A}}^{\prime}\right)^{2}+\hat{x}_{2}^{2}\left(g_{\mathrm{V}}^{\prime}+g_{\mathrm{A}}^{\prime}\right)^{2}\right], \\
& F^{-+}\left(\hat{x}_{1}, \hat{x}_{2}\right)=\frac{G_{\mathrm{F}}^{2} m_{\mathrm{W}}^{4} \hat{s}\left(g_{\mathrm{V}}+g_{\mathrm{A}}\right)^{2}}{8 \pi\left(m_{\mathrm{V}}^{2}-\hat{s}^{2}\right.} \\
& \quad \times\left[\left(1-\hat{x}_{2}\right)^{2}\left(g_{\mathrm{V}}^{\prime}+g_{\mathrm{A}}^{\prime}\right)^{2}+\hat{x}_{2}^{2}\left(g_{\mathrm{V}}^{\prime}-g_{\mathrm{A}}^{\prime}\right)^{2}\right] .
\end{aligned}
$$

(Note that the factor 8 appears in the denominator rather than 32 due to the fact that we don't average over initial spins here.) Using the appropriate polarized quark functions, one can now calculate the desired polarized inclusive cross sections.

\section{A. 7. Resonance decay angular distributions}

In this subsection we summarize the density-matrix structure for the decays of resonances with $J \leqslant \frac{3}{2}$. The general results are given in sect. 3 of ref. [42]. For $J=1$ the decay angular distribution is:

$$
\begin{aligned}
& W_{1}(\theta, \varphi)=\frac{3}{4 \pi}\left\{\cos ^{2} \theta \rho_{00}+\frac{1}{2} \sin ^{2} \theta\left(\rho_{11}+\rho_{-1-1}\right)\right. \\
& \left.-\sin ^{2} \theta \operatorname{Re}\left(\rho_{1-1} \mathrm{e}^{2 i \varphi}\right)-\sqrt{\frac{1}{2}} \sin 2 \theta \operatorname{Re}\left(\rho_{10} \mathrm{e}^{i \varphi}-\rho_{-10} \mathrm{e}^{-i \varphi}\right)\right\} \\
& \sim \ldots+\sin ^{2} \theta \sin 2 \varphi \operatorname{Im} \rho_{1-1}+\sqrt{\frac{1}{2}} \sin 2 \theta \sin \varphi\left(\operatorname{Im} \rho_{10}+\operatorname{Im} \rho_{-10}\right),
\end{aligned}
$$


where the parity-violating terms are exhibited in the last line. The angles $\theta, \varphi$ are the polar and azimuthal angles of one of the decay products (in a two-body decay) in the rest frame of the resonance, with respect to some quantization axis. If parity were conserved in the production of the resonance its density matrix would satisfy

$$
\rho_{m m^{\prime}}=(-1)^{m-m^{\prime}} \rho_{-m-m^{\prime}}
$$

for a $z$-axis in the production plane. Then one would have $\operatorname{Im} \rho_{10}=-\operatorname{Im} \rho_{-10}$ and $\operatorname{Im} \rho_{1-1}=0$ and the terms of interest to us would vanish. In general for weak production they are not zero, and the amount of parity violation in the production is measured by the expectation value of $\sin 2 \theta \sin \varphi$, or of $\sin ^{2} \theta \sin 2 \varphi$,

either

$\langle\sin 2 \theta \sin \varphi\rangle \neq 0 \Rightarrow$ parity violation,

and/or

$$
\left\langle\sin ^{2} \theta \sin 2 \varphi\right\rangle \neq 0 \Rightarrow \text { parity violation. }
$$

For a resonance with a three-body decay, the angles $\theta, \varphi$ are measured with respect to the normal to the plane in which the three particles lie.

(One word of caution is needed. Parity conservation in the production of the resonance only implies eq. (A.42) when no other direction is available, i.e., when the initial particles are unpolarized, no spins are observed in the other outgoing particles, and no cuts are made which could define a direction (e.g., don't look for a resonance plus a $\mathrm{K}^{0}$ in some upper half plane). General parity relations are given in ref. [43].

For $J=\frac{3}{2}$ the analogous relation is:

$$
\begin{aligned}
& W_{3 / 2}(\theta, \varphi)=\frac{3}{4 \pi}\left\{\frac{1}{2}\left(\frac{1}{3}+\cos ^{2} \theta\right)\left(\rho_{1 / 2,1 / 2}+\rho_{-1 / 2,-1 / 2}\right)\right. \\
& \quad+\frac{1}{2} \sin ^{2} \theta\left(\rho_{3 / 2,3 / 2}+\rho_{-3 / 2,-3 / 2}\right)-\sqrt{\frac{1}{3}} \sin 2 \theta \operatorname{Re}\left(\rho_{3 / 2,1 / 2} \mathrm{e}^{i \varphi}\right. \\
& \left.\left.-\rho_{-3 / 2,-1 / 2} \mathrm{e}^{-i \varphi}\right)-\sqrt{\frac{1}{3}} \sin ^{2} \theta \operatorname{Re}\left(\rho_{3 / 2,-1 / 2} \mathrm{e}^{2 i \varphi}+\rho_{-3 / 2,1 / 2} \mathrm{e}^{-2 i \varphi}\right)\right\} \\
& \sim \ldots+\sqrt{\frac{1}{3}} \sin 2 \theta \sin \varphi\left(\operatorname{Im} \rho_{3 / 2,1 / 2}+\operatorname{Im} \rho_{-3 / 2,-1 / 2}\right) \\
& \quad+\sqrt{\frac{1}{3}} \sin ^{2} \theta \sin 2 \varphi\left(\operatorname{Im} \rho_{3 / 2,-1 / 2}-\operatorname{Im} \rho_{-3 / 2,1 / 2}\right),
\end{aligned}
$$

and the signals for parity violation in the production are again those given in eq. (A.43)

For spins 1 and $\frac{3}{2}$ above, only the interference of helicity $0\left( \pm \frac{1}{2}\right)$ with the parityviolating difference of helicity $\pm 1\left( \pm \frac{3}{2}\right)$ states can be measured with the parity-conserving resonance decay. For spin $\frac{1}{2}$ we can use the parity-violating $\Lambda$ decay as well. The distribution is

$$
W_{1 / 2}(\theta, \varphi)=N\left\{1+\alpha \cos \theta p_{z}+\alpha \sin \theta \cos \varphi p_{x}+\alpha \sin \theta \sin \varphi p_{y}\right\},
$$


Table 8

Detection of parity violation

\begin{tabular}{lll}
\hline Spin $J$ & Decay & Terms \\
\hline$\frac{1}{2}$ & PV & $\cos \theta$ \\
& & $\sin \theta \cos \varphi$ \\
$1, \frac{3}{2}$ & PC & $\sin 2 \theta \sin \varphi$ \\
& & $\sin ^{2} \theta \sin 2 \varphi$ \\
\hline
\end{tabular}

Consider a spin- $J$ resonance decaying into particles with decay angles $\theta, \varphi$. In order to identify parity violation in the production of the resonance one must measure a non-zero expectation value for one of the expressions given above. The decay of the resonance may be parity conserving $(\mathrm{PC})$ or parity violating (PV).

where

$$
\begin{aligned}
& p_{z}=\rho_{1 / 2,1 / 2}-\rho_{-1 / 2,-1 / 2}, \\
& p_{x}=2 \operatorname{Re} \rho_{1 / 2,-1 / 2}, \\
& p_{y}=-2 \operatorname{Im} \rho_{1 / 2,-1 / 2},
\end{aligned}
$$

and parity conservation in the production would give $p_{z}=p_{x}=0$. Thus observing either

$$
\begin{aligned}
& \langle\cos \theta\rangle \neq 0, \\
& \text { or } \quad\langle\sin \theta \cos \varphi\rangle \neq 0,
\end{aligned}
$$

requires parity violation in the production. The $\cos \theta$ effect will be most easy to interpret theoretically as it measures the difference in rate of production of helicity $\frac{1}{2}$ and helicity $-\frac{1}{2}$ states, without a dependence on phases.

To summarize, detection of the terms in table 8 in inclusive resonance production would show that the production had a parity-violating contribution. Presumably, as argued in sect. 2 of the paper, this can be interpreted as due to a scattering via an intermediate vector boson, and thus a signal which can demonstrate the existence of such a particle, and/or allow us to study its properties. More generally, it is a signal of some weak-interaction process with a relatively large cross section.

\section{References}

[1] C. Quigg, Rev. Mod. Phys. 49 (1977) 297.

[2] F. Halzen, Phys. Rev. D15 (1977) 1929;

R.F. Peierls, T.L. Trueman and L.-L. Wang, Phys. Rev. D16 (1977) 1397;

L.B. Okun and M.B. Voloshin, Nucl. Phys. B120 (1977) 459. 
[3] R.W. Brown, K.O. Mikaelian and Mary K. Gaillard, Nucl. Phys. B75 (1974) 112;

M. Perrottet, Harvard University preprint, HUTP-77/A048 (1977).

[4] E.M. Henley, Proc. 1974 Summer Studies on high-energy physics with polarized beams.

[5] G.W. Look and E. Fischbach, Phys. Rev. D16 (1977) 1369.

[6] A.D. Krisch, private communication.

[7] M.J. Alguard et al., Phys. Rev. Lett. 37 (1976) 1261.

[8] R.T. Ross, A study of the reaction $\nu \mathrm{p} \rightarrow \mu^{-} \pi^{+} \mathrm{p}$, Proc. of Neutrinos 78 Conf., Purdue University, April 1978.

[9] A. Astbury et al., CERN/SPSC/78-06 (1978).

[10] J.K. Walker (ed.), Colliding-beam physics at Fermilab, 1977 summer study.

[11] S.M. Berman, J.D. Bjorken and J.B. Kogut, Phys. Rev. D4 (1971) 3388.

[12] S.D. Ellis and M.B. Kislinger, Phys. Rev. D9 (1974) 2027.

[13] E. Fischbach and G.W. Look, Phys. Rev. D15 (1977) 2576.

[14] R.D. Field and R.P. Feynman, Phys. Rev. D15 (1977) 2590.

[15] R.D. Field, Phys. Rev. Lett. 40 (1978) 997; private communication.

[16] D. Antreasyan et al., Phys. Rev. Lett. 38 (1977) 112.

[17] G. Jancso et al., Nucl. Phys. B124 (1977) 1.

[18] C.W. Akerlof et al., Phys. Rev. Lett. 39 (1977) 861.

[19] W.R. Ditzler et al., Phys. Lett. 71B (1977) 451;

C.W. Akerlof and R. Thun, private communication.

[20] M. Antinucci et al., Nuovo Cim. Lett. 6 (1973) 121.

[21] D. Antreasyan et al., Phys. Rev. Lett. 38 (1977) 115.

[22] R.N. Mohapatra and D.P. Sidhu, Phys. Rev. D16 (1977) 2843.

[23] M.A.B. Beg et al., Phys. Rev. Lett. 38 (1977) 1252.

[24] Proc. 1977 Ann Arbor Workshop on possibilities for higher energy polarized proton beams (ed. A.D. Krisch and A.J. Salthouse), AIP Conf. Proc. (1978).

[25] K.H. Craig, Nucl. Phys. B109 (1976) 156.

[26] S.D. Drell and T.-M. Yan, Phys. Rev. Lett. 25 (1970) 316.

[27] I.P. Auer et al., Phys. Lett. 70B (1977) 475.

[28] H.E. Haber and G.L. Kane, Nucl. Phys. B129 (1977) 429.

[29] H.E. Haber and G.L. Kane, Nucl. Phys. B144 (1978) 525.

[30] T. Appelquist and J.D. Bjorken, Phys. Rev. D4 (1971) 3726;

M. Veltman, Phys. Lett. 70B (1977) 253;

B.W. Lee, C. Quigg and H.B. Thacker, Phys. Rev. Lett. 38 (1977) 883.

[31] H.E. Haber, Doctoral thesis, University of Michigan (1978), unpublished.

[32] S. Weinberg, Phys. Rev. Lett. 19 (1967) 1264;

A. Salam, in Elementary particle theory, ed. V. Svartholm (Almquist and Wiksell, Stockholm, 1968), p. 367.

[33] M. Holder et al., Phys. Lett. 71B (1977) 222.

[34] V. Barger and R.J.N. Phillips, Nucl. Phys. B73 (1974) 269;

R. Carlitz, Phys. Lett. 58B (1975) 345.

[35] R. Carlitz, and J. Kaur, Phys. Rev. Lett. 38 (1977) 673.

[36] G.W. Look and E. Fischbach, Phys. Rev. D16 (1977) 211.

[37] J. Kaur, Nucl. Phys. B128 (1977) 219.

[38] F.E. Close and D. Sivers, Phys. Rev. Lett. 39 (1977) 1116.

[39] R.D. Field and R.P. Feynman, Nucl. Phys. B136 (1978) 1.

[40] A.P. Contogouris, R. Gaskell and A. Nicolaidis, Phys. Rev. D17 (1978) 839.

[41] J. Burger, talk at the XIII Annual Rencontre de Moriond, Les Arcs, France, March 1978.

[42] J.D. Jackson, lectures given at Les Houches 1965, ed. C. DeWitt and M. Jacob (Gordon and Breach, New York, 1965).

[43] G.R. Goldstein and J.F. Owens, Nucl. Phys. B103 (1976) 145.

[44] F.W. Busser et al., Nucl. Phys. B106 (1976) 1. 